\title{
Quasi-morphisms on contactomorphism groups and contact rigidity
}

\author{
Matthew Strom Borman* and Frol Zapolsky ${ }^{\dagger}$
}

November 7, 2018

Dedicated with gratitude to our teacher Leonid Polterovich on his 50th birthday

\begin{abstract}
We build homogeneous quasi-morphisms on the universal cover of the contactomorphism group for certain prequantizations of monotone symplectic toric manifolds. This is done using Givental's nonlinear Maslov index and a contact reduction technique for quasimorphisms. We show how these quasi-morphisms lead to a hierarchy of rigid subsets of contact manifolds. We also show that the nonlinear Maslov index has a vanishing property, which plays a key role in our proofs. Finally we present applications to orderability of contact manifolds and Sandon-type metrics on contactomorphism groups.
\end{abstract}

\section{Introduction and results}

\subsection{Quasi-morphisms on contactomorphism groups}

A quasi-morphism on a group $G$ is a function $\mu: G \rightarrow \mathbb{R}$ which is a homomorphism up to a bounded error, that is there is $D>0$ such that

$$
|\mu(a b)-\mu(a)-\mu(b)| \leq D \text { for all } a, b \in G,
$$

and it is homogeneous if $\mu\left(a^{k}\right)=k \mu(a)$ for all $a \in G$ and $k \in \mathbb{Z}$. It is straightforward to show that homogeneous quasi-morphisms are conjugation-invariant and restrict to homomorphisms on abelian subgroups. See [Bav91, Cal09, Kot04 for background on quasi-morphisms, their connection with bounded cohomology, and their applications to commutator length and other quantitative group-theoretic questions. For the sake of exposition, in this paper by quasimorphism we will mean a non-zero, homogeneous quasi-morphism.

The construction and applications of quasi-morphisms on infinite-dimensional groups of symmetries have recently been a popular theme of research [Ent04, FOOO11b, GG04, Ghy01, Ghy07, Pol06, She11, Ush11. One reason is that groups of diffeomorphisms are often perfect [Ban97], and thus admit no non-zero homomorphisms to $\mathbb{R}$ and so one is led to study quasi-morphisms on them instead. When the group has an interesting metric such as the hydrodynamic metric on the group of volume-preserving diffeomorphisms of a Riemannian

\footnotetext{
*Partially supported by NSF grants DMS-1006610 and DMS-1304252.

${ }^{\dagger}$ Partially supported by Deutsche Forschungsgemeinschaft grant DFG/CI 45/5-1 while at LMU (Munich).
} 
manifold [Bra12, BS13] or Hofer's metric on the Hamiltonian group of a symplectic manifold [EP03, Py06], quasi-morphisms can be used to understand the coarse geometry of these groups. Another reason is that oftentimes quasi-morphisms on the symmetry groups of symplectic and contact manifolds lead to results on the geometry of the underlying manifolds themselves, which is also the case in the present paper.

We will only consider contact manifolds $(V, \xi)$ where $V$ is connected and closed, unless stated otherwise, and $\xi$ is a cooriented contact structure. We will write $(V, \xi, \alpha)$ if we want to specify a choice of a coorienting contact form $\alpha$ such that $\xi=\operatorname{ker} \alpha$. The Reeb vector field associated to a contact form $\alpha$ will be denoted $R_{\alpha}$ and is uniquely defined by

$$
\alpha\left(R_{\alpha}\right)=1 \quad \text { and } \quad \iota_{R_{\alpha}} d \alpha=0 .
$$

Let $\operatorname{Cont}_{0}(V, \xi)$ be the identity component of the group of contactomorphisms and denote by $\widetilde{\operatorname{Cont}}_{0}(V, \xi)$ its universal cover.

Given a smooth time-dependent function $h:[0,1] \times V \rightarrow \mathbb{R}$, called a contact Hamiltonian, there is a unique time-dependent vector field $\left\{X_{h_{t}}\right\}_{t \in[0,1]}$ satisfying

$$
\alpha\left(X_{h_{t}}\right)=h_{t} \quad \text { and } \quad d \alpha\left(X_{h_{t}}, \cdot\right)=-d h_{t}+d h_{t}\left(R_{\alpha}\right) \alpha \quad \text { where } h_{t}=h(t, \cdot) .
$$

The vector field $\left\{X_{h_{t}}\right\}$ preserves $\xi$ and integrates into a contact isotopy denoted $\left\{\phi_{h}^{t}\right\}_{t \in[0,1]}$. This establishes a bijection, depending on the contact form $\alpha$, between smooth functions $h:[0,1] \times V \rightarrow \mathbb{R}$ and contact isotopies based at the identity id of $V$. If $h, g \in C^{\infty}(V)$, then

$$
\{h, g\}_{\alpha}:=-d g\left(X_{h}\right)+d h\left(R_{\alpha}\right) g
$$

is the contact Hamiltonian corresponding to the Lie bracket of $X_{h}$ and $X_{g}$. We write $\widetilde{\phi}_{h}$ for the element of $\widetilde{\operatorname{Cont}}_{0}(V, \xi)$ represented by the contact isotopy $\left\{\phi_{h}^{t}\right\}_{t \in[0,1]}$. For the constant function $h=1$, the vector field $X_{1}=R_{\alpha}$ is the Reeb vector field and hence $\widetilde{\phi}_{1}$ is the element generated by the Reeb flow.

Following Eliashberg-Polterovich [EP00] for $\widetilde{\phi} \in{\widetilde{\operatorname{Cont}_{0}}}_{0}(V, \xi)$ we will write id $\preceq \widetilde{\phi}$ if there is a nonnegative contact Hamiltonian $h$ such that $\widetilde{\phi}=\widetilde{\phi}_{h}$ in $\widetilde{\operatorname{Cont}}_{0}(V, \xi)$. The nonnegativity of $h$ is equivalent to $X_{h_{t}}$ being nowhere negatively transverse to $\xi$, and therefore it is independent of $\alpha$. This induces a reflexive and transitive relation on $\widetilde{\operatorname{Cont}}_{0}(V, \xi)$ where

$$
\widetilde{\phi} \preceq \widetilde{\psi} \text { if and only if } \text { id } \preceq \widetilde{\phi}^{-1} \widetilde{\psi}
$$

which is also bi-invariant [EP00]. The contact manifold $(V, \xi)$ is called orderable if $\preceq$ is a partial order on $\widetilde{\operatorname{Cont}}_{0}(V, \xi)$, that is $\preceq$ is also anti-symmetric.

Definition 1.1. For a quasi-morphism $\mu$ : $\widetilde{\operatorname{Cont}}_{0}(V, \xi) \rightarrow \mathbb{R}$, define the following properties:

(i) Monotone: $\widetilde{\phi} \preceq \widetilde{\psi}$ implies $\mu(\widetilde{\phi}) \leq \mu(\widetilde{\psi})$.

(ii) $C^{0}$-continuous: If $h$ is a smooth contact Hamiltonian and there is a sequence of smooth contact Hamiltonians $h^{(n)}$ such that $h^{(n)} \rightarrow h$ in $C^{0}([0,1] \times V)$, then $\mu\left(\widetilde{\phi}_{h^{(n)}}\right) \rightarrow \mu\left(\widetilde{\phi}_{h}\right)$.

(iii) Vanishing: If $U \subset V$ is an open subset and there is $\psi \in \operatorname{Cont}_{0}(V, \xi)$ with $\psi(U) \cap U=\emptyset$, then $\mu\left(\widetilde{\phi}_{h}\right)=0$ for all contact Hamiltonians $h$ with $\operatorname{supp}(h) \subset[0,1] \times U$.

In general, a subset $S \subset V$ is displaceable if there is $\psi \in \operatorname{Cont}_{0}(V, \xi)$ with $\psi(S) \cap \bar{S}=\emptyset$. Note that the vanishing property is independent of the choice of a contact form. 


\subsubsection{Givental's asymptotic nonlinear Maslov index}

Besides Poincaré's rotation number on $\widetilde{\operatorname{Cont}}_{0}\left(S^{1}\right) \equiv \widetilde{\operatorname{Diff}}_{0}\left(S^{1}\right)$, the only previous construction of quasi-morphisms on contactomorphism groups was Givental's asymptotic nonlinear Maslov index [Giv90, Section 9]

$$
\mu_{\mathrm{Giv}}: \widetilde{\operatorname{Cont}}_{0}\left(\mathbb{R} P^{2 d-1}\right) \rightarrow \mathbb{R}
$$

with $\mathbb{R} P^{2 d-1}$ being taken with the standard contact structure. Results in Giv90, Section 9] imply $\mu_{\text {Giv }}$ is a homogeneous quasi-morphism, as Ben Simon [BS07, Theorem 0.2] proved. In Section 4.3 we will review the definition and relevant properties of Givental's quasi-morphism, and prove the following proposition.

Proposition 1.2. Givental's quasi-morphism $\mu_{\mathrm{Giv}}$ : $\widetilde{\operatorname{Cont}}_{0}\left(\mathbb{R} P^{2 d-1}\right) \rightarrow \mathbb{R}$ is (i) monotone, (ii) $C^{0}$-continuous, and (iii) has the vanishing property.

For time-independent contact Hamiltonians Givental proved Giv90, Corollary 3, Section 9] that $\mu_{\text {Giv }}$ is monotone and $C^{0}$-continuous, and as we will explain his proofs work in general. The vanishing property, which does not appear in [Giv90], together with Theorem 1.19 below give an alternative proof of Ben Simon's [BS07, Theorem 0.6].

\subsubsection{Quasi-morphisms for prequantizations of even toric manifolds}

A prequantization of a symplectic manifold $(M, \omega)$ is a contact manifold $(V, \xi, \alpha)$ with a map $\pi:(V, \alpha) \rightarrow(M, \omega)$ defining a principal $S^{1}$-bundle such that $\pi^{*} \omega=d \alpha$, and the Reeb vector field $R_{\alpha}$ induces the free $S^{1}$-action on $V$ where $S^{1}=\mathbb{R} / \hbar \mathbb{Z}, \hbar>0$ being the minimal period of a closed Reeb orbit.

A toric symplectic manifold $\left(M^{2 n}, \omega, \mathbb{T}\right)$ is a symplectic manifold endowed with an effective Hamiltonian action of a torus $\mathbb{T}$ of dimension $n$. The action is induced by a moment map $M \rightarrow \mathfrak{t}^{*}$, where $\mathfrak{t}^{*}$ is the dual of the Lie algebra $\mathfrak{t}$ of $\mathbb{T}$, and the image of the moment map is called the moment polytope and denoted $\Delta$. If $\Delta$ has $d$ facets, then it is given by

$$
\Delta=\left\{x \in \mathfrak{t}^{*} \mid\left\langle\nu_{j}, x\right\rangle+a_{j} \geq 0 \text { for } j=1, \ldots, d\right\},
$$

where the conormals $\nu_{j}$ are primitive vectors in the integer lattice $\mathfrak{t}_{\mathbb{Z}}:=\operatorname{ker}(\exp : \mathfrak{t} \rightarrow \mathbb{T})$.

A symplectic manifold $(M, \omega)$ is monotone if and only if there is a positive constant $\lambda>0$ so that $[\omega]=\lambda c_{1}(M) \in H^{2}(M ; \mathbb{R})$, and for toric manifolds this is equivalent to being able to choose the moment map so that $a_{1}=\cdots=a_{d}=\lambda$. We call the moment polytope $\Delta$ even if $\sum_{j=1}^{d} \nu_{j} \in 2 \mathfrak{t}_{\mathbb{Z}}$ and we say that a toric manifold is even if its associated moment polytope is even. In Section 1.6 we give examples of closed monotone even symplectic toric manifolds. We can now formulate our main result.

Theorem 1.3. Every closed monotone even toric symplectic manifold $(M, \omega, \mathbb{T})$ has a prequantization $(\widehat{M}, \xi, \alpha)$ for which there is a quasi-morphism

$$
\mu: \widetilde{\operatorname{Cont}}_{0}(\widehat{M}, \xi) \rightarrow \mathbb{R}
$$

that is monotone, has the vanishing property, and is $C^{0}$-continuous. 
In Section 1.3 below we discuss the significance of this theorem in the context of stable Calabi quasi-morphisms on the universal cover of the Hamiltonian group of a symplectic manifold.

Theorem 1.8 below shows how a monotone quasi-morphism on $\widetilde{\operatorname{Cont}_{0}}(V)$ can induce a monotone quasi-morphism on $\widetilde{\operatorname{Cont}}_{0}(\bar{V})$ if $(\bar{V}, \bar{\xi})$ is the result of performing contact reduction on $(V, \xi)$. In Section 2.1 we will show how the even moment polytope of a monotone toric manifold $(M, \omega, \mathbb{T})$ naturally leads to a prequantization $(\widehat{M}, \xi, \alpha)$ obtained from $\mathbb{R} P^{2 d-1}$ via contact reduction. The proof of Theorem 1.3 is then given in Section 2.2 where we apply Theorem 1.8 to Givental's quasi-morphism $\mu_{\text {Giv }}$ on $\widetilde{\operatorname{Cont}}_{0}\left(\mathbb{R} P^{2 d-1}\right)$ to build the monotone quasi-morphisms $\mu$ : $\widetilde{\operatorname{Cont}}_{0}(\widehat{M}, \xi) \rightarrow \mathbb{R}$.

Not all prequantizations $\pi:(V, \alpha) \rightarrow(M, \omega)$ of a monotone even toric symplectic manifold $(M, \omega)$ admit non-trivial monotone quasi-morphisms on $\widetilde{\operatorname{Cont}}_{0}(V)$. This is because if $V$ is not orderable, then there is no monotone quasi-morphism on $\widetilde{\operatorname{Cont}}_{0}(V)$ (see Theorem 1.28 below). The basic example is the standard contact sphere $S^{2 d-1}$ for $d \geq 2$, which is a prequantization of the even toric manifold $\mathbb{C} P^{d-1}$ but is not orderable. See Section 1.5.1 for further discussion about orderability and quasi-morphisms.

Remark 1.4. If $\pi:(V, \alpha) \rightarrow(M, \omega)$ is a prequantization, then for the subgroup $\mathbb{Z}_{k} \leq S^{1}$ the quotient manifold $V / \mathbb{Z}_{k}$ is also a prequantization of $M$. Pulling back contact Hamiltonians via the projection $V \rightarrow V / \mathbb{Z}_{k}$ induces a homomorphism $\widetilde{\operatorname{Cont}_{0}}\left(V / \mathbb{Z}_{k}\right) \rightarrow \widetilde{\operatorname{Cont}_{0}}(V)$ and therefore the quasi-morphisms of Theorem 1.3 give rise to quasi-morphisms on $\widetilde{\operatorname{Cont}}_{0}\left(\widehat{M} / \mathbb{Z}_{k}\right)$.

Remark 1.5. There is work in progress by Karshon-Pabiniak-Sandon [KPS13] to generalize Givental's construction of the asymptotic nonlinear Maslov index, with lens spaces being the first step. If for a prime $p$ there is a monotone quasi-morphism with the vanishing property

$$
\mu_{\mathrm{KPS}}: \widetilde{\operatorname{Cont}}_{0}\left(S^{2 d-1} / \mathbb{Z}_{p}\right) \rightarrow \mathbb{R}
$$

where $\mathbb{Z}_{p}$ acts by multiplication by a $p$-th root of unity, then Theorem 1.3 would generalize to the closed monotone toric symplectic manifolds $(M, \omega, \mathbb{T})$ whose sum of conormals in the moment polytope satisfies $\sum_{j=1}^{d} \nu_{j} \in p \cdot \mathfrak{t}_{\mathbb{Z}}$ (see the proof of Lemma 2.1).

\subsubsection{Reduction for quasi-morphisms on contactomorphism groups}

In [Bor12, Bor13] a procedure for pushing forward quasi-morphisms on the universal cover of the Hamiltonian group of a symplectic manifold via symplectic reduction was developed. In this paper we will streamline this technique and adapt it to the contact setting in Theorem 1.8 . which will be used to prove Theorem 1.3 . Before we can formulate the reduction theorem for quasi-morphisms, we need the following two definitions.

Definition 1.6. For a contact manifold $(V, \xi, \alpha)$, a closed submanifold $Y \subset V$ transverse to $\xi$ is strictly coisotropic with respect to $\alpha$ if it is coisotropic, that is the subbundle $T Y \cap \xi$ of the symplectic vector bundle $\left(\left.\xi\right|_{Y}, d \alpha\right)$ is coisotropic:

$$
\left\{X \in \xi_{y} \mid \iota_{X} d \alpha=0 \text { on } T_{y} Y \cap \xi_{y}\right\} \subset T_{y} Y \cap \xi_{y} \quad \text { for all } y \in Y,
$$

and additionally $R_{\alpha}(y) \in T_{y} Y$ for all $y \in Y$, that is the Reeb vector field is tangent to $Y$. 
The property of being coisotropic is independent of the contact form and, assuming transversality, being strictly coisotropic with respect to $\alpha$ is equivalent to

$$
T_{y} Y^{d \alpha}:=\left\{X \in T_{y} V \mid \iota_{X} d \alpha=0 \text { on } T_{y} Y\right\} \subset T_{y} Y \quad \text { for all } y \in Y .
$$

One can check that $Y \subset(V, \xi)$ is strictly coisotropic with respect to some contact form if and only if $Y$ is the diffeomorphic image of a coisotropic submanifold under the projection $S V \rightarrow V$ where $S V$ is the symplectization of $V$.

Definition 1.7. Let $\mu$ : $\widetilde{\operatorname{Cont}}_{0}(V, \xi) \rightarrow \mathbb{R}$ be a monotone quasi-morphism. A closed subset 1 ) $Y \subset V$ is $\mu$-subheavy if

$$
\mu\left(\widetilde{\phi}_{h}\right)=0
$$

whenever $h$ is an autonomous contact Hamiltonian with $\left.h\right|_{Y}=0$.

Here now is the reduction theorem for quasi-morphisms on contactomorphism groups, which we will prove in Section 3 . Consider the setting

$$
(V, \xi, \alpha) \supset\left(Y,\left.\alpha\right|_{Y}\right) \stackrel{\rho}{\longrightarrow}(\bar{V}, \bar{\xi}, \bar{\alpha})
$$

where $(V, \xi, \alpha)$ and $(\bar{V}, \bar{\xi}, \bar{\alpha})$ are closed contact manifolds, $Y \subset V$ is a closed submanifold that is strictly coisotropic with respect to $\alpha$, and $\rho: Y \rightarrow \bar{V}$ is a fiber bundle such that $\rho^{*} \bar{\alpha}=\left.\alpha\right|_{Y}$.

Theorem 1.8. In the setting (1.9) if $Y \subset V$ is subheavy for a monotone quasi-morphism $\mu: \widetilde{\operatorname{Cont}}_{0}(V, \xi) \rightarrow \mathbb{R}$, then it induces a monotone quasi-morphism

$$
\bar{\mu}: \widetilde{\operatorname{Cont}}_{0}(\bar{V}, \bar{\xi}) \rightarrow \mathbb{R} \quad \text { defined by } \bar{\mu}\left(\widetilde{\phi}_{\bar{h}}\right):=\mu\left(\widetilde{\phi}_{h}\right)
$$

where $h \in C^{\infty}([0,1] \times V)$ is any contact Hamiltonian such that $\left.h\right|_{[0,1] \times Y}=\rho^{*} \bar{h}$. The vanishing property and $C^{0}$-continuity passes from $\mu$ to $\bar{\mu}$.

An example of (1.9) is given by contact reduction [Gei97, Theorem 6] where a compact Lie group $G$ acts on $V$ preserving $\alpha$ with moment map $P: V \rightarrow \mathfrak{g}^{*}$. In this case $Y=P^{-1}(0)$ is strictly coisotropic with respect to $\alpha$ and $\bar{V}=Y / G$ is a contact manifold assuming $G$ acts freely on $Y$. When we prove Theorem 1.3 in Section 2.2 it will be in the case of contact reduction for torus actions on $\mathbb{R} P^{2 d-1}$.

It should be noted that, considering more general group actions on $\mathbb{R} P^{2 d-1}$, it is possible to construct monotone quasi-morphisms with the vanishing property on prequantizations of symplectic manifolds more general than toric ones, however we shall not pursue this direction here.

\subsection{Contact rigidity}

Nondisplaceability phenomena in contact manifolds is one aspect of contact rigidity and it is much less studied than nondisplaceability in symplectic manifolds by Hamiltonian diffeomorphisms ABM14, AM13a, BEP04, Cho04, EP06, EP09b, FOO009, McD11, WW13, Woo11. As with the symplectic setting, contact nondisplaceability goes back to a conjecture of Arnold that for the standard contact structure on the jet space $J^{1} N=T^{*} N \times \mathbb{R}$ of a closed

\footnotetext{
${ }^{1)}$ See Remark 1.16 regarding the closed assumption, which also applies to the definition of superheavy below.
} 
manifold $N$, the zero section $\{(q, 0,0) \mid q \in N\}$ cannot be displaced from the zero wall $\{(q, 0, z) \mid q \in N, z \in \mathbb{R})\}$ by a contact isotopy and this was proved by Chekanov [Che96] using generating functions. Using spectral invariants from generating functions Zap13 proved contact rigidity for smooth and singular subsets of the standard contact $T^{*} N \times S^{1}$. Floer theoretic methods have also been used by Eliashberg-Hofer-Salamon [EHS95] and Ono [Ono96] to detect nondisplaceable submanifolds in unit cotangent bundles of closed manifolds and in certain prequantizations. Recently sheaf-theoretic methods have been also been playing a role in symplectic and contact rigidity, see for example [Tam08, GKS12].

In the series of papers [BEP04, EP03, EP06, EP09b] Entov-Polterovich showed how to use the machinery of their quasi-morphisms on the universal cover of the Hamiltonian group of a symplectic manifold $(M, \omega)$ and quasi-states in order to study the rigidity of symplectic intersections. In particular in [EP09b] they showed that there is a hierarchy of rigid subsets in symplectic manifolds for which they introduced the terminology of heavy and superheavy subsets.

\subsubsection{Superheavy and subheavy sets for monotone quasi-morphisms on $\widetilde{\text { Cont }_{0}}$}

Inspired by Entov-Polterovich's work, in this paper we will show how monotone quasimorphisms on $\widetilde{\operatorname{Cont}}_{0}(V)$ can also be used to study the rigidity of intersections in contact manifolds. In analogy to the terms heavy and superheavy for subsets of symplectic manifolds, we will also show how such monotone quasi-morphisms detect a hierarchy of rigid subsets in contact manifolds, namely subheavy (defined above) and superheavy sets:

Definition 1.9. If $\mu$ : $\widetilde{\operatorname{Cont}}_{0}(V, \xi) \rightarrow \mathbb{R}$ is a monotone quasi-morphism, then a closed subset $Y \subset V$ is $\mu$-superheavy if

$$
\mu\left(\widetilde{\phi}_{h}\right)>0
$$

for all autonomous contact Hamiltonians $h \in C^{\infty}(V)$ such that $\left.h\right|_{Y}>0$.

Given a prequantization $\pi:(V, \alpha) \rightarrow(M, \omega)$, in Section 1.3 we will discuss how superheavy subsets in the symplectic manifold $(M, \omega)$ are related to subheavy and superheavy subsets of the contact manifold $(V, \alpha)$. The basic properties of superheavy sets in contact manifolds are given by the following proposition.

Proposition 1.10. Let $\mu$ : ${\widetilde{\operatorname{Cont}_{0}}}_{0}(V, \xi) \rightarrow \mathbb{R}$ be a monotone quasi-morphism.

(i) The properties $\mu$-superheavy and $\mu$-subheavy are independent of the choice of contact form $\alpha$ for $\xi$ used to link contact Hamiltonians and contact isotopies.

(ii) If $Z$ is $\mu$-superheavy ( $\mu$-subheavy) and $Z \subset Y$, then $Y$ is $\mu$-superheavy ( $\mu$-subheavy).

(iii) The property of being $\mu$-subheavy is preserved by elements of $\operatorname{Cont}_{0}(V, \xi)$, and likewise for $\mu$-superheavy.

(iv) The entire manifold $V$ is $\mu$-superheavy.

This next theorem and its corollary relates subheavy and superheavy sets with contact rigidity.

Theorem 1.11. Let $\mu$ : $\widetilde{\operatorname{Cont}}_{0}(V, \xi) \rightarrow \mathbb{R}$ be a monotone quasi-morphism. 
(i) All $\mu$-superheavy subsets are $\mu$-subheavy.

(ii) If $Y$ is $\mu$-superheavy and $Z$ is $\mu$-subheavy, then $Y \cap Z \neq \emptyset$.

As an immediate corollary of Proposition 1.10(iii) and Theorem 1.11 we have:

Corollary 1.12. If $Y \subset V$ is $\mu$-subheavy and $Z \subset V$ is $\mu$-superheavy for a monotone quasimorphism $\mu$ : $\widehat{\operatorname{Cont}}_{0}(V, \xi) \rightarrow \mathbb{R}$, then the following holds:

(i) $Y$ cannot be displaced from $Z$, that is $\psi(Y) \cap Z \neq \emptyset$ for all $\psi \in \operatorname{Cont}_{0}(Y)$.

(ii) $Z$ is nondisplaceable, that is $\psi(Z) \cap Z \neq \emptyset$ for all $\psi \in \operatorname{Cont}_{0}(Y)$.

See Section 4.1 for the proofs of Proposition 1.10 and Theorem 1.11, which together with Corollary 1.12 are analogous to the basic properties of heavy and superheavy subsets of a symplectic manifold [EP09b, Section 1.4]. We also have the following criterion for when a $\mu$-subheavy set is automatically $\mu$-superheavy, which we prove in Section 4.1 .

Proposition 1.13. Let $\mu$ : ${\widetilde{\mathrm{Cont}_{0}}}_{0}(V, \xi) \rightarrow \mathbb{R}$ be a monotone quasi-morphism and $Y \subset V$ be a $\mu$-subheavy subset. If $Y$ is preserved by the flow of some positive contact vector field, then $Y$ is $\mu$-superheavy.

In the context of Theorem 1.8 , note that Proposition 1.13 implies that the $\mu$-subheavy subset $Y \subset V$, which is strictly coisotropic, is actually $\mu$-superheavy.

As the next theorem shows, the properties of being subheavy and superheavy are respected by the reduction of the quasi-morphisms in Theorem 1.8. Recall in Theorem 1.8 one has contact manifolds $(V, \xi, \alpha)$ and $(\bar{V}, \bar{\xi}, \bar{\alpha})$ and a closed submanifold $Y \subset V$ with a fiber bundle $\rho: Y \rightarrow \bar{V}$. There are monotone quasi-morphisms

$$
\mu: \widetilde{\operatorname{Cont}}_{0}(V, \xi) \rightarrow \mathbb{R} \text { and } \bar{\mu}: \widetilde{\operatorname{Cont}_{0}}(\bar{V}, \bar{\xi}) \rightarrow \mathbb{R}
$$

where by definition $\bar{\mu}\left(\widetilde{\phi}_{\bar{h}}\right):=\mu\left(\widetilde{\phi}_{h}\right)$ for $h \in C^{\infty}([0,1] \times V)$ being any contact Hamiltonian that satisfies $\rho^{*} \bar{h}=\left.h\right|_{[0,1] \times Y}$.

Theorem 1.14. For monotone, $C^{0}$-continuous quasi-morphisms 1.11) from Theorem 1.8, if $Z \subset V$ is $\mu$-subheavy, then $\rho(Y \cap Z) \subset \bar{V}$ is $\bar{\mu}$-subheavy and likewise for superheavy sets.

Proof. First note that since $Y$ is $\mu$-superheavy by Proposition 1.13 there is a non-trivial intersection $Y \cap Z \neq \emptyset$ by Theorem 1.11 if $Z$ is $\mu$-subheavy.

Assume $Z \subset V$ is $\mu$-superheavy and let $\bar{h} \in C^{\infty}(\bar{V})$ be such that $\left.\bar{h}\right|_{\rho(Y \cap Z)}>0$. For $\epsilon>0$ sufficiently small, let $\bar{f} \in C^{\infty}(\bar{V})$ be such that $\bar{f}=\epsilon$ in a neighborhood of $\rho(Y \cap Z)$ and $\bar{h} \geq \bar{f}$. Now we can pick an extension $f \in C^{\infty}(V)$ so that $\left.f\right|_{Z}=\epsilon$ and $\rho^{*} \bar{f}=\left.f\right|_{Y}$. Since $Z$ is $\mu$-superheavy it follows that $\bar{\mu}\left(\widetilde{\phi}_{\bar{f}}\right)=\mu\left(\widetilde{\phi}_{f}\right)>0$, and hence by monotonicity $\bar{\mu}\left(\widetilde{\phi}_{\bar{h}}\right)>0$. Therefore $\rho(Y \cap Z) \subset \bar{V}$ is $\bar{\mu}$-superheavy.

Assume $Z \subset V$ is $\mu$-subheavy and let $\bar{h} \in C^{\infty}(\bar{V})$ be such that $\left.\bar{h}\right|_{\rho(Y \cap Z)}=0$. Pick a sequence $\bar{f}_{n} \in C^{\infty}(\bar{V})$ such that there is $C^{0}$-convergence $\bar{f}_{n} \rightarrow \bar{h}$ and there are neighborhoods $\mathcal{N}_{n}$ of $\rho(Y \cap Z)$ such that $\left.\bar{f}_{n}\right|_{\mathcal{N}_{n}}=0$. We can pick extensions $f_{n} \in C^{\infty}(V)$ so that $\left.f_{n}\right|_{Z}=0$ and $\rho^{*} \bar{f}_{n}=\left.f_{n}\right|_{Y}$. Since $Z$ is $\mu$-subheavy it follows that $\bar{\mu}\left(\widetilde{\phi}_{\bar{f}_{n}}\right):=\mu\left(\widetilde{\phi}_{f_{n}}\right)=0$ and hence $\bar{\mu}\left(\widetilde{\phi}_{h}\right)=0$ since $\bar{\mu}$ is $C^{0}$-continuous. Therefore $\rho(Y \cap Z) \subset \bar{V}$ is $\bar{\mu}$-subheavy. 
Remark 1.15. We did not use the assumption of $C^{0}$-continuity of $\mu$ to prove that superheaviness descends under reduction. Also the descent for subheaviness holds without the $C^{0}$-continuity assumption if $Z$ intersects $Y$ sufficiently nicely, for instance if there is a small tubular neighborhood pr: $U \rightarrow Y$ of $Y$ such that pr $\left.\right|_{U \cap Z}: U \cap Z \rightarrow Y \cap Z$ is a fiber bundle. However in general it is not possible to find a smooth extension $h$ of $\rho^{*} \bar{h}$ with $\left.h\right|_{Z}=0$, which we get around by using the $C^{0}$-continuity assumption.

Remark 1.16. We only consider closed subsets in the hierarchy of subheavy and superheavy subsets, and for instance we use this assumption in our proof of Theorem 1.11 and Proposition 1.13. Of course it is possible to extend the definitions and the theorems to arbitrary subsets via closure, but we have suppressed this for the sake of exposition.

\subsubsection{Rigid Legendrians and pre-Lagrangians}

As demonstrated by previous work in contact rigidity [Eli91, EHS95, Ono96, EP00] two important classes of submanifolds in contact manifolds are Legendrians and pre-Lagrangians. Recall [EHS95, Section 2.2] that a pre-Lagrangian submanifold $Y^{n+1} \subset\left(V^{2 n+1}, \xi\right)$ is one such that $Y$ is transverse to $\xi$ and there is a contact form $\alpha$ such that $\left.d \alpha\right|_{Y}=0$, that is $Y$ is a strictly coisotropic submanifold of minimal dimension. An equivalent definition from EHS95, Proposition 2.2.2] is that $Y$ is the diffeomorphic image of a Lagrangian under the projection $S V \rightarrow V$ where $S V$ is the symplectization of $V$. A nice class of examples is as follows: for a prequantization $\pi:(V, \alpha) \rightarrow(M, \omega)$ and a Lagrangian $L \subset M$, the submanifold $\pi^{-1}(L) \subset V$ is pre-Lagrangian. Note that Proposition 1.13 implies every closed subheavy pre-Lagrangian submanifold is superheavy.

As we will see from our examples of subheavy and superheavy subsets of contact manifolds in Section 1.4, prototypically a subheavy submanifold is a Legendrian and a superheavy submanifold is a pre-Lagrangian. In particular in Corollary 1.26 we explicitly identify a $\mu$ subheavy Legendrian submanifold and a $\mu$-superheavy pre-Lagrangian torus for each of the quasi-morphisms in Theorem 1.3. For the case of Givental's quasi-morphism on $\mathbb{R} P^{2 d-1}$, a $\mu_{\text {Giv-subheavy Legendrian is }}$

$$
\mathbb{R} P_{L}^{d-1}:=\left\{[z] \in \mathbb{R} P^{2 d-1} \mid z \in \mathbb{R}^{d}\right\}
$$

and a $\mu_{\mathrm{Giv}}$-superheavy pre-Lagrangian torus is

$$
T_{\mathbb{R} P}:=\left\{\left.[z] \in \mathbb{R} P^{2 d-1}|| z_{1}\right|^{2}=\cdots=\left|z_{d}\right|^{2}=1 / \pi\right\},
$$

where we are viewing $\mathbb{R} P^{2 d-1}$ as the quotient of the sphere $S^{2 d-1} \subset \mathbb{C}^{d}$ with radius $\sqrt{d / \pi}$. See Lemmas 1.23 and 1.22 for the proofs.

More generally we have the following existence theorem for nondisplaceable pre-Lagrangian tori, analogous to Entov-Polterovich's proof [EP06, Theorem 2.1] of the existence of nondisplaceable Lagrangians in closed toric symplectic manifolds.

Theorem 1.17. If $\mu$ : ${\widetilde{\operatorname{Cont}_{0}}}_{0}(V, \xi) \rightarrow \mathbb{R}$ is a monotone quasi-morphism with the vanishing property and $(V, \alpha)$ is a prequantization of a closed toric manifold $(M, \omega)$, then $V$ contains a nondisplaceable pre-Lagrangian torus.

See Section 4.1 for the proof of Theorem 1.17 . 


\subsection{Quasi-morphisms on $\widetilde{\operatorname{Ham}}(M)$ and symplectic quasi-states}

For a closed symplectic manifold $(M, \omega)$, a smooth Hamiltonian $F:[0,1] \times M \rightarrow \mathbb{R}$ induces a time-dependent vector field $\left\{X_{F_{t}}\right\}_{t \in[0,1]}$ by

$$
\iota_{X_{F_{t}}} \omega=-d F_{t} \quad \text { where } F_{t}=F(t, \cdot) .
$$

Integrating $X_{F_{t}}$ gives a Hamiltonian isotopy $\left\{\phi_{F}^{t}\right\}_{t \in[0,1]}$ of $M$ based at id the identity of $M$ and these are in bijection with smooth Hamiltonians $F:[0,1] \times M \rightarrow \mathbb{R}$ normalized so $\int_{M} F_{t} \omega^{n}=0$ for all $t \in[0,1]$. The Hamiltonian group $\operatorname{Ham}(M)$ is the set of time-one maps $\phi_{F}^{1}$ of such Hamiltonian isotopies and $\widetilde{\operatorname{Ham}}(M)$ is its universal cover. We write $\widetilde{\phi}_{H}$ for the element of $\widetilde{\operatorname{Ham}}(M)$ represented by the Hamiltonian isotopy $\left\{\phi_{H}^{t}\right\}_{t \in[0,1]}$. For normalized functions $H, G \in C^{\infty}(M)$ their Poisson bracket

$$
\{H, G\}_{\omega}:=\omega\left(X_{G}, X_{H}\right)=-d G\left(X_{H}\right)
$$

is the Hamiltonian whose vector field is the Lie bracket of $X_{H}$ and $X_{G}$. A subset $S \subset M$ is displaceable if there is $\phi \in \operatorname{Ham}(M)$ so that $\phi(S) \cap \bar{S}=\emptyset$.

For a quasi-morphism $\mu_{M}: \widetilde{\operatorname{Ham}}(M) \rightarrow \mathbb{R}$ one defines the following two properties [EP03, EPZ07:

(i) Stable: For normalized Hamiltonians $H, G:[0,1] \times M \rightarrow \mathbb{R}$

$$
\int_{0}^{1} \min _{M}\left(H_{t}-G_{t}\right) d t \leq \frac{\mu_{M}\left(\widetilde{\phi}_{G}\right)-\mu_{M}\left(\widetilde{\phi}_{H}\right)}{\operatorname{Vol}(M)} \leq \int_{0}^{1} \max _{M}\left(H_{t}-G_{t}\right) d t
$$

where $\operatorname{Vol}(M)=\int_{M} \omega^{n}$.

(ii) Calabi: If $U \subset M$ is an open displaceable subset and if $H:[0,1] \times M \rightarrow \mathbb{R}$ has support in $[0,1] \times U$, then

$$
\mu_{M}\left(\widetilde{\phi}_{H}\right)=\operatorname{Cal}_{U}\left(\widetilde{\phi}_{H}\right):=\int_{0}^{1} \int_{U} H_{t} \omega^{n} d t
$$

Such quasi-morphisms were constructed by Entov-Polterovich in [EP03] using spectral invariants in Hamiltonian Floer theory and their construction has been refined and extended in [EP08, FOOO11b, Lan11, Lan13a, Lan13b, MVZ12, Ost06, Ush11].

On a closed symplectic manifold $(M, \omega)$ a quasi-state is a functional $\zeta: C^{\infty}(M) \rightarrow \mathbb{R}$ satisfying the following properties for all $H, K \in C^{\infty}(M)$ :

(i) Monotone: If $H \leq K$, then $\zeta(H) \leq \zeta(K)$.

(ii) Normalized: $\zeta(1)=1$.

(iii) Quasi-linearity: If $\{H, K\}_{\omega}=0$, then $\zeta(H+K)=\zeta(H)+\zeta(K)$.

These quasi-states are the symplectic version of Aarnes' notion of topological quasi-state Aar91. As established in [EP06, every stable quasi-morphism $\mu_{M}: \widehat{\operatorname{Ham}}(M) \rightarrow \mathbb{R}$ induces a quasi-state $\zeta_{\mu_{M}}: C^{\infty}(M) \rightarrow \mathbb{R}$ defined by

$$
\zeta_{\mu_{M}}(H):=\frac{\int_{M} H \omega^{n}-\mu_{M}\left(\widetilde{\phi}_{H}\right)}{\operatorname{Vol}(M)}
$$


Such quasi-states are $\operatorname{Ham}(M)$-invariant. If $\mu_{M}$ also has the Calabi property, then $\zeta_{\mu_{M}}$ has the vanishing property, that is $\zeta(H)=0$ whenever $\operatorname{supp}(H) \subset M$ is displaceable.

Definition 1.18. Let $\zeta: C^{\infty}(M) \rightarrow \mathbb{R}$ be a quasi-state on a closed symplectic manifold $(M, \omega)$. A closed subset $X \subset M$ is $\zeta$-superheavy if

$$
\min _{X} H \leq \zeta(H) \leq \max _{X} H
$$

for all $H \in C^{\infty}(M)$.

This definition was introduced in EP09b and $\zeta$-superheavy sets $X \subset M$ are nondisplaceable when $\zeta$ is $\operatorname{Ham}(M)$-invariant, by [EP09b, Theorem 1.4]. See [BEP04, BEP12, EP06, EP09a, EP09b, EP10, EPP12, EPZ07, FOOO11b, Kha09 for various applications of EntovPolterovich's quasi-morphisms and quasi-states.

Recall for a prequantization $\pi:(V, \alpha) \rightarrow(M, \omega)$ one has the following central extension of Lie algebras

$$
0 \rightarrow \mathbb{R} \rightarrow\left(C^{\infty}(V)^{S^{1}},\{\cdot, \cdot\}_{\alpha}\right) \rightarrow\left(C^{\infty}(M) / \mathbb{R},\{\cdot, \cdot\}_{\omega}\right) \rightarrow 0 .
$$

Here $C^{\infty}(V)^{S^{1}} \simeq C^{\infty}(M)$ is the set of $S^{1}$-invariant functions on $V$ and $C^{\infty}(M) / \mathbb{R}$ is canonically the Lie algebra of $\operatorname{Ham}(M)$. When $M$ is closed this sequence has a unique splitting by the Lie algebra homomorphism

$$
\sigma: C^{\infty}(M) / \mathbb{R} \rightarrow C^{\infty}(V)^{S^{1}} \quad \text { given by } \quad H \mapsto \pi^{*} H-\frac{\int_{M} H \omega^{n}}{\operatorname{Vol}(M)}
$$

and $\sigma$ induces a homomorphism

$$
\pi^{*}: \widetilde{\operatorname{Ham}}(M) \rightarrow \widetilde{\operatorname{Cont}_{0}}(V) \text { where } \pi^{*}\left(\widetilde{\phi}_{H}\right)=\widetilde{\phi}_{\sigma(H)} .
$$

See [BS10, Section 1.3] for more details on this point and in particular a proof that (1.17) is a homomorphism.

We now have the following result, generalizing Ben Simon [BS07], which uses the homomorphism (1.17) to relate quasi-morphisms on $\widetilde{\operatorname{Cont}}_{0}$ and $\widetilde{\text { Ham. }}$. Recall that $\widetilde{\phi}_{1} \in \widetilde{\operatorname{Cont}}_{0}(V)$ is the element generated by the Reeb vector field $R_{\alpha}$.

Theorem 1.19. Let $\pi:(V, \alpha) \rightarrow(M, \omega)$ be a prequantization of a closed symplectic manifold and let $\mu: \widetilde{\operatorname{Cont}}_{0}(V) \rightarrow \mathbb{R}$ be a monotone quasi-morphism, then

$$
\mu_{M}:=-\frac{\operatorname{Vol}(M)}{\mu\left(\widetilde{\phi}_{1}\right)}\left(\mu \circ \pi^{*}\right): \widetilde{\operatorname{Ham}}(M) \rightarrow \mathbb{R}
$$

is a stable quasi-morphism. The quasi-state associated to $\mu_{M}$ from 1.15 has the form:

$$
\zeta_{\mu_{M}}(H):=\frac{\mu\left(\widetilde{\phi}_{\pi^{*} H}\right)}{\mu\left(\widetilde{\phi}_{1}\right)} .
$$

If $\mu$ has the vanishing property, then $\mu_{M}$ has the Calabi property and $\zeta_{\mu_{M}}$ has the vanishing property. 
A historical remark is in order. While Givental [Giv90] applied his quasi-morphism to various contact rigidity phenomena on $\mathbb{R} P^{2 d-1}$, such as the existence of Reeb chords, it was first in the symplectic setting that Entov-Polterovich developed a systematic approach to use their quasi-morphisms in order to study symplectic rigidity. However as Theorem 1.19 shows, for prequantizable symplectic manifolds, quasi-morphisms on $\widetilde{\text { Cont }}_{0}$ are potentially more fundamental objects than quasi-morphisms on Ham. A related question is if it is possible to obtain one of Entov-Polterovich's quasi-morphisms on $\widehat{\operatorname{Ham}}(M)$ from a quasi-morphism on $\widetilde{\operatorname{Cont}}_{0}(V)$ via Theorem 1.19 , and this is open even for the case of the prequantization $\mathbb{R} P^{3} \rightarrow \mathbb{C} P^{1}$.

The following proposition shows how the Entov-Polterovich notion of superheaviness (1.16) with respect to a symplectic quasi-state on $(M, \omega)$ is related to sub- and superheaviness with respect to a quasi-morphism on $\widetilde{\operatorname{Cont}_{0}}(V)$ when $\pi:(V, \alpha) \rightarrow(M, \omega)$ is a prequantization.

Proposition 1.20. If $\pi:(V, \alpha) \rightarrow(M, \omega)$ is a prequantization, $\mu: \widetilde{\operatorname{Cont}_{0}}(V) \rightarrow \mathbb{R}$ is a monotone quasi-morphism, and $\mu_{M}: \widetilde{\operatorname{Ham}}(M) \rightarrow \mathbb{R}$ is the quasi-morphism induced according to Theorem 1.19, then

(i) if $Y \subset V$ is $\mu$-subheavy, then $\pi(Y) \subset M$ is $\zeta_{\mu_{M}}$-superheavy;

(ii) if $X \subset M$ is $\zeta_{\mu_{M}}$-superheavy, then $\pi^{-1}(X) \subset V$ is $\mu$-superheavy.

Theorem 1.19 and Proposition 1.20 are proved in Section 4.2 .

Given a collection of $\left(H_{1}, \ldots, H_{k}\right)$ pairwise Poisson commuting Hamiltonians on $M$, organized as a map $\Phi: M \rightarrow \mathbb{R}^{k}$, Entov-Polterovich in [EP06] defined a fiber $\Phi^{-1}(p)$ to be a stem if every other fiber $\Phi^{-1}(q) \subset M$ was displaceable. They proved in EP09b, Theorem 1.8] that a stem $X \subset M$ is superheavy with respect to any quasi-state with the vanishing property. Using Theorem 1.19 and Proposition 1.20 we now have the following corollary for any prequantization $\pi:(\widehat{M, \alpha}) \rightarrow(M, \omega)$ and monotone quasi-morphism $\mu$ : $\widehat{\operatorname{Cont}}_{0}(\widehat{M}) \rightarrow \mathbb{R}$ with the vanishing property:

Corollary 1.21. If $X \subset(M, \omega)$ is a stem, then $\pi^{-1}(X) \subset \widehat{M}$ is $\mu$-superheavy.

Stems can be very singular subsets, an example being the product of 1-skeletons of fine triangulations of 2-spheres [EP06, Corollary 2.5].

\subsection{Examples of contact rigidity}

In this subsection we will present concrete examples of subheavy and superheavy subsets of contact manifolds.

\subsubsection{Examples using Givental's quasi-morphism}

We will start with the rigidity results that just use Givental's monotone quasi-morphism $\mu_{\text {Giv }}: \widetilde{\text { Cont }}_{0}\left(\mathbb{R} P^{2 d-1}\right) \rightarrow \mathbb{R}$. For us it will be convenient to introduce the following models of the standard contact $S^{2 d-1}$ and $\mathbb{R} P^{2 d-1}$. For $\gamma=\left(\gamma_{1}, \ldots, \gamma_{d}\right) \in \mathbb{N}^{d}$, consider the sphere

$$
S_{\gamma}^{2 d-1}=\left\{\left.z \in \mathbb{C}^{d}\left|\pi \sum_{j=1}^{d} \gamma_{j}\right| z_{j}\right|^{2}=\sum_{j=1}^{d} \gamma_{j}\right\}
$$


with the contact form given by the restriction of

$$
\alpha_{\text {std }}=\frac{1}{2} \sum_{j=1}^{d}\left(x_{j} d y_{j}-y_{j} d x_{j}\right)
$$

to $S_{\gamma}^{2 d-1}$ with Reeb flow

$$
\phi_{R_{\gamma}}^{t}\left(z_{1}, \ldots, z_{d}\right)=\left(e^{2 \pi i \gamma_{1} t / d} z_{1}, \ldots, e^{2 \pi i \gamma_{d} t / d} z_{d}\right) .
$$

For the antipodal $\mathbb{Z}_{2}$-action on $\mathbb{C}^{d}$, let

$$
\left(\mathbb{R} P_{\gamma}^{2 d-1}, \xi_{\gamma}\right):=\left(S_{\gamma}^{2 d-1} / \mathbb{Z}_{2}, \text { ker } \alpha_{\text {std }}\right) .
$$

Note when $\gamma=(1, \ldots, 1)$ that $\left(\mathbb{R} P_{\gamma}^{2 d-1}, \xi_{\gamma}\right)$ is the standard model for $\left(\mathbb{R} P^{2 d-1}, \xi\right)$, so we will drop the reference to $\gamma$ in this case. Via radial projection $z \mapsto \frac{\sqrt{d}}{\sqrt{\pi}} \frac{z}{|z|}$, which induces a contactomorphism

$$
r:\left(\mathbb{R} P_{\gamma}^{2 d-1}, \xi_{\gamma}\right) \rightarrow\left(\mathbb{R} P^{2 d-1}, \xi\right),
$$

we have Givental's quasi-morphism $\mu_{\text {Giv }}: \widetilde{\operatorname{Cont}_{0}}\left(\mathbb{R} P_{\gamma}^{2 d-1}\right) \rightarrow \mathbb{R}$ for any $\gamma \in \mathbb{N}^{d}$.

Lemma 1.22. The torus

$$
T_{\mathbb{R} P}:=\left\{\left.[z] \in \mathbb{R} P_{\gamma}^{2 d-1}|| z_{1}\right|^{2}=\cdots=\left|z_{d}\right|^{2}=1 / \pi\right\} \subset \mathbb{R} P_{\gamma}^{2 d-1}
$$

is $\mu_{\mathrm{Giv}}$-superheavy.

Proof of Lemma 1.22. Since the radial projection $(1.23)$ preserves $T_{\mathbb{R} P}$, it suffices to show $T_{\mathbb{R} P} \subset \mathbb{R} P^{2 d-1}$ is $\mu_{\text {Giv }}$-superheavy. Consider the prequantization $\pi: \mathbb{R} P^{2 d-1} \rightarrow \mathbb{C} P^{d-1}$ where we take

$$
\mathbb{C} P^{d-1}=\left\{\left.\left[z_{1}: \cdots: z_{d}\right]\left|\pi \sum\right| z_{j}\right|^{2}=d\right\} .
$$

Using the Hamiltonian $U(d)$-action on $\mathbb{C} P^{d-1}$, the Clifford torus $\mathbb{T}_{\text {Clif }}^{d-1}:=\pi\left(T_{\mathbb{R} P}\right)$ can be shown to be a stem [BEP04, Lemma 5.1]. Since $\mu_{\text {Giv }}$ has the vanishing property by Proposition 1.2 , it follows from Corollary 1.21 that $T_{\mathbb{R} P}$ is $\mu_{\text {Giv }}$-superheavy.

Lemma 1.22 will play a large role in our proof of Theorem 1.3 for it will ensure we are applying Theorem 1.8 to a $\mu_{\mathrm{Giv}}$-superheavy subset.

While by Theorem 1.11 it is impossible for a Legendrian submanifold to be superheavy, since they are always displaceable (for instance by an arbitrarily small positive contact isotopy), it is possible for a Legendrian to be subheavy as the next example shows. The proof is given in Section 4.3 .

Lemma 1.23. The standard Legendrian

$$
\mathbb{R} P_{L}^{d-1}:=\left\{[z] \in \mathbb{R} P_{\gamma}^{2 d-1} \mid z \in \mathbb{R}^{d}\right\} \subset \mathbb{R} P_{\gamma}^{2 d-1}
$$

is $\mu_{\mathrm{Giv}}$-subheavy.

Once we take the orbit of $\mathbb{R} P_{L}^{d-1}$ under the Reeb flow, which is a closed subset since the Reeb flow is periodic, we get the following immediate corollary of Lemma 1.23 and Proposition 1.13 . 
Corollary 1.24. The subset

$$
L_{\gamma}:=\bigcup_{t \in \mathbb{R}} \phi_{R_{\gamma}}^{t}\left(\mathbb{R} P_{L}^{d-1}\right) \subset\left(\mathbb{R} P_{\gamma}^{2 d-1}, \xi_{\gamma}\right)
$$

is $\mu_{\mathrm{Giv}}$-superheavy.

Corollary 1.24 can be used to prove rigidity in weighted complex projective spaces. Recall for a primitive vector $\gamma \in \mathbb{N}^{d}$ that the weighted complex projective space $\mathbb{C} P(\gamma)$ is the symplectic orbifold obtained as the quotient of $S_{\gamma}^{2 d-1}$ by the Reeb flow (1.21). A Hamiltonian isotopy of $\mathbb{C} P(\gamma)$ is by definition an isotopy that lifts to a contact isotopy of $S_{\gamma}^{2 d-1}$ preserving the contact form. If the fixed point set of the involution on $\mathbb{C} P(\gamma)$ induced by complex conjugation on $\mathbb{C}^{d}$ is

$$
\mathbb{R} P(\gamma) \subset \mathbb{C} P(\gamma)
$$

and

$$
T_{\mathbb{C} P}:=\left\{\left.[z] \in \mathbb{C} P(\gamma)|| z_{1}\right|^{2}=\cdots=\left|z_{d}\right|^{2}=1 / \pi\right\} \subset \mathbb{C} P(\gamma)
$$

then we have the following proposition.

Proposition 1.25. If for a primitive vector $\gamma=\left(\gamma_{1}, \ldots, \gamma_{d}\right) \in \mathbb{N}^{d}$ each $\gamma_{j}$ is odd, then

$$
\mathbb{R} P(\gamma) \cap \psi(\mathbb{R} P(\gamma)) \neq \emptyset, \quad \mathbb{R} P(\gamma) \cap \psi\left(T_{\mathbb{C} P}\right) \neq \emptyset, \quad T_{\mathbb{C} P} \cap \psi\left(T_{\mathbb{C} P}\right) \neq \emptyset
$$

for all Hamiltonian isotopies $\psi$ of $\mathbb{C} P(\gamma)$.

Proof. The fact that all $\gamma_{j}$ are odd is equivalent to the time $t=\frac{1}{2}$ Reeb flow (1.21) being the antipodal map on $S_{\gamma}^{2 d-1}$. Therefore if each $\gamma_{j}$ is odd, then the quotient map $S_{\gamma}^{2 d-1} \rightarrow \mathbb{C P}(\gamma)$ factors through the projection map

$$
\pi: \mathbb{R} P_{\gamma}^{2 d-1} \rightarrow \mathbb{C} P(\gamma)
$$

and hence any Hamiltonian isotopy of $\mathbb{C} P(\gamma)$ lifts to a contact isotopy of $\mathbb{R} P_{\gamma}^{2 d-1}$.

By the definitions, under the projection map $\pi\left(L_{\gamma}\right) \subset \mathbb{R} P(\gamma)$ and $\pi\left(T_{\mathbb{R} P}\right)=T_{\mathbb{C} P}$. Since $L_{\gamma}$ and $T_{\mathbb{R} P}$ are $\mu_{\mathrm{Giv}}$-superheavy by Lemmas 1.24 and 1.22 it follows from Theorem 1.11 that both $L_{\gamma}$ and $T_{\mathbb{R} P}$ are nondisplaceable and cannot be displaced from each other by a contact isotopy. Therefore the same holds for $\mathbb{R} P(\gamma)$ and $T_{\mathbb{C} P}$ for Hamiltonian isotopies.

Nondisplaceability of $T_{\mathbb{C} P} \subset \mathbb{C} P(\gamma)$ for any primitive $\gamma \in \mathbb{N}^{d}$ was proved by Woodward Woo11 and Cho-Poddar CP12. Nondisplaceability of $\mathbb{R} P(\gamma)$ for an odd primitive vector $\gamma$ was previously proved by $\mathrm{Lu}[\mathrm{Lu} 08]$.

\subsubsection{Examples using the quasi-morphisms from Theorem 1.3}

In the proof of Theorem 1.3 in Section 2 we will apply Theorem 1.8 to Givental's quasimorphism to build $\mu$. In particular for an appropriate primitive vector $\gamma \in \mathbb{N}^{d}$ in Section 2.2 we present the prequantization $(\widehat{M}, \alpha)$ in the setting 1.9$)$ of Theorem 1.8

$$
\left(\mathbb{R} P_{\gamma}^{2 d-1}, \xi_{\gamma}, \alpha_{\text {std }}\right) \supset\left(Y,\left.\alpha_{\text {std }}\right|_{Y}\right) \stackrel{\rho}{\longrightarrow}(\widehat{M}, \xi, \alpha)
$$


where $Y$ is a $\mu_{\text {Giv }}$-superheavy submanifold containing $T_{\mathbb{R} P}$ and $\mu:=\bar{\mu}_{\text {Giv }}$ is the reduction of Givental's quasi-morphism. For the torus $T_{\mathbb{R} P}$ and standard Legendrian $\mathbb{R} P_{L}^{d-1}$ in $\mathbb{R} P_{\gamma}^{2 d-1}$ from 1.24 and 1.25 define the following two subsets of $\widehat{M}$

$$
T_{\widehat{M}}:=\rho\left(T_{\mathbb{R} P}\right) \quad \text { and } \quad \widehat{M}_{\mathbb{R}}:=\rho\left(Y \cap \mathbb{R} P_{L}^{d-1}\right) .
$$

Note that $T_{\widehat{M}}$ is a pre-Lagrangian torus while $\widehat{M}_{\mathbb{R}}$ is Legendrian. We now have the following corollary.

Corollary 1.26. For a quasi-morphism $\mu$ : $\widetilde{\operatorname{Cont}}_{0}(\widehat{M}) \rightarrow \mathbb{R}$ from Theorem 1.3 , the preLagrangian $T_{\widehat{M}}$ is $\mu$-superheavy and the Legendrian $\widehat{M}_{\mathbb{R}}$ is $\mu$-subheavy.

Proof. This follows from Theorem 1.14 together with Lemma 1.22 and Lemma 1.23.

The next result concerns rigidity for the real part $M_{\mathbb{R}} \subset(M, \omega)$ of a symplectic toric manifold, which is characterized as the fixed point set of the anti-symplectic involution that preserves the moment map. Using the prequantization $\pi:(\widehat{M}, \alpha) \rightarrow(M, \omega)$ we construct in Section 2.1 for a monotone even toric manifold, the real part of $M$ can be identified with

$$
M_{\mathbb{R}}:=\pi\left(\widehat{M}_{\mathbb{R}}\right)
$$

where $\widehat{M}_{\mathbb{R}} \subset \widehat{M}$ is from $(1.28)$. For the quasi-morphism $\mu$ : $\widetilde{\operatorname{Cont}}_{0}(\widehat{M}) \rightarrow \mathbb{R}$ from Theorem 1.3 . let $\zeta_{\mu_{M}}: C^{\infty}(M) \rightarrow \mathbb{R}$ be the induced symplectic quasi-state on $(M, \omega)$ from Theorem 1.19 .

Proposition 1.27. The real part $M_{\mathbb{R}} \subset(M, \omega)$ of a monotone even toric symplectic manifold is $\zeta_{\mu_{M}}$-superheavy and hence nondisplaceable.

Proof. Using $\widehat{M}_{\mathbb{R}}$ is $\mu$-subheavy by Corollary 1.26 it follows $M_{\mathbb{R}}=\pi\left(\widehat{M}_{\mathbb{R}}\right)$ is $\zeta_{\mu_{M}}$-superheavy by Proposition 1.20 and therefore is nondisplaceable.

Haug [Hau13] proved the nondisplaceability part of Proposition 1.27 without the even assumption using Biran-Cornea's Lagrangian quantum homology [BC09a, BC09b].

Similarly the central toric fiber $T_{M} \subset(M, \omega)$ of a monotone even toric manifold is nondisplaceable and cannot be displaced from the real part $M_{\mathbb{R}}$. This is because $\pi^{-1}\left(T_{M}\right)=T_{\widehat{M}}$ so Proposition 1.20 and Corollary 1.26 imply $T_{M}$ is $\zeta_{\mu_{M}}$-superheavy. The nondisplaceability now follows from [EP09b, Theorem 1.4]. These results have been established by various authors AM13a, AA12, Cho08, EP06, FOOO11a. In particular Abreu-Macarini AM13a, showed how simple previous nondisplaceability results in $\mathbb{C} P^{n}$ can be combined with symplectic reduction to prove the nondisplaceability results for $T_{M}$ and the pair $\left(T_{M}, M_{\mathbb{R}}\right)$, but could not prove $M_{\mathbb{R}}$ was nondisplaceable.

\subsection{Orderability and metrics on $\widetilde{\operatorname{Cont}}_{0}$}

Recall from Section 1.1 that a contact manifold $(V, \xi)$ is orderable if $\widetilde{\operatorname{Cont}}_{0}(V, \xi)$ is partially ordered by the relation $\preceq$ from (1.4). 


\subsubsection{Orderability for contact manifolds and quasi-morphisms}

There has been a fair amount of research concerning orderability of contact manifolds. Since we are mainly dealing with closed contact manifolds, let us give examples of orderable and non-orderable closed contact manifolds. Eliashberg-Kim-Polterovich prove in EKP06] that the ideal contact boundary of a sufficiently subcritical Weinstein manifold is not orderable. In particular the standard contact spheres $S^{2 d-1}$ are not orderable for $d \geq 2$. Cosphere bundles of closed manifolds are known to be orderable [AF13, CN10, EKP06, EP00] and more generally Albers-Merry proved in AM13b] that Liouville-fillable contact manifolds with nonvanishing Rabinowitz Floer homology are orderable. Using the connection between orderability and contact squeezing developed by Eliashberg-Kim-Polterovich [EKP06], Milin [Mil08] and Sandon [San11b] proved that lens spaces are orderable.

In [EP00, Section 1.3.E] Eliashberg-Polterovich proved that $\mathbb{R} P^{2 d-1}$ is orderable using Givental's quasimorphism $\mu_{\text {Giv }}$. Their argument works in general and implies the following.

Theorem 1.28 ([EP00]). A contact manifold $(V, \xi)$ is orderable if there is a monotone quasimorphism $\mu$ on $\widetilde{\operatorname{Cont}}_{0}(V, \xi)$.

Proof. By [EP00, Criterion 1.2.C] to prove $(V, \xi)$ is orderable it suffices to prove id $\neq \widetilde{\phi}_{h}$ in $\widetilde{\operatorname{Cont}}_{0}(V, \xi)$ for any contact Hamiltonian with $h>0$ on $[0,1] \times V$. Since $\mu(\mathrm{id})=0$, we are done because for any such contact Hamiltonian $\mu\left(\widetilde{\phi}_{h}\right)>0$ by Proposition 1.10 (iv).

Corollary 1.29. The contact manifolds $(\widehat{M}, \xi)$ in Theorem 1.3 are orderable.

Recall that the contact manifolds $(\widehat{M}, \xi)$ are obtained from contact reduction of $\mathbb{R} P^{2 d-1}$, which is of course orderable. It would be interesting to prove Corollary 1.29 directly, that is to prove orderability persists under contact reduction.

By Theorem 1.28, orderability is a necessary condition for the existence of a non-zero homogeneous monotone quasi-morphism on $\widetilde{\operatorname{Cont}_{0}}(V)$. However in general the converse is not well understood and potentially is a delicate question, which we will illustrate with the following examples regarding $\mathbb{R}^{2 n} \times S^{1}$ and its group of compactly supported contactomorphisms Cont ${ }_{0}^{c}\left(\mathbb{R}^{2 n} \times S^{1}\right)$, where the contact form is $\alpha_{\text {std }}+d t$ and $d t$ is the angular form on $S^{1}=\mathbb{R} / \mathbb{Z}$.

Example 1.30. Sandon has proved San10, San11a] that $\mathbb{R}^{2 n} \times S^{1}$ is orderable, that is the Eliashberg-Polterovich relation $(1.4)$ is indeed a partial order on the group $\widetilde{\operatorname{Cont}_{0}^{c}}\left(\mathbb{R}^{2 n} \times S^{1}\right)$, and also proved it induces a partial order on $\operatorname{Cont}_{0}^{c}\left(\mathbb{R}^{2 n} \times S^{1}\right)$. However $\operatorname{Cont}_{0}^{c}\left(\mathbb{R}^{2 n} \times S^{1}\right)$ admits no non-zero homogeneous quasi-morphisms, due to a general argument of Kotschick [Kot08, Theorem 4.2] and the fact that Cont $_{0}^{c}$ is always perfect due to Rybicki Ryb10].

Example 1.31. This example rests on the speculation that $\widetilde{\operatorname{Cont}}_{0}^{c}$ is in general perfect. In this case again Kotschick's argument proves $\widetilde{\operatorname{Cont}_{0}^{c}}\left(\mathbb{R}^{2 n} \times S^{1}\right)$ admits no non-zero homogeneous quasi-morphisms, despite $\preceq$ being a partial order on $\widetilde{\operatorname{Cont}_{0}^{c}}\left(\mathbb{R}^{2 n} \times S^{1}\right)$.

Example 1.32. Consider now the domain $B_{R}^{2 n} \times S^{1}$ where

$$
B_{R}^{2 n}:=\left\{\left.z \in \mathbb{C}^{n}|\pi| z\right|^{2}<R\right\} .
$$


Since $\mathbb{R}^{2 n} \times S^{1}$ is contactomorphic to $B_{1}^{2 n} \times S^{1}$ by [EKP06, Proposition 1.24], Example 1.31 indicates $\widetilde{\operatorname{Cont}_{0}^{c}}\left(B_{1}^{2 n} \times S^{1}\right)$ does not admit a non-zero homogeneous quasi-morphism.

On the other hand, $\widetilde{\operatorname{Cont}}_{0}^{c}\left(B_{R}^{2 n} \times S^{1}\right)$ admits a non-zero homogeneous quasi-morphism whenever $\frac{2 n}{n+1}<R<2$. When $R<2$ we have the contact embedding

$$
\Phi: B_{R}^{2 n} \times S^{1} \rightarrow \mathbb{R} P^{2 n+1} \quad \text { by } \quad(z, t) \mapsto e^{\pi i t} \sqrt{\frac{n+1}{2}}\left(z, \sqrt{\frac{2}{\pi}-|z|^{2}}\right)
$$

written as a map to $S^{2 n+1}=\left\{\left.z \in \mathbb{C}^{n+1}|\pi| z\right|^{2}=n+1\right\}$ from $(1.19)$; where $t \in[0,1)$. When $R>\frac{2 n}{n+1}$, one can check the image $\Phi\left(B_{R}^{2 n}\right)$ contains the $\mu_{\mathrm{Giv}}$-superheavy torus $T_{\mathbb{R} P} \subset \mathbb{R} P^{2 n+1}$ from Lemma 1.22 and hence one can use $\Phi$ to pull-back $\mu_{\text {Giv }}$ to a non-zero homogeneous quasimorphism on $\operatorname{Cont}_{0}^{c}\left(B_{R}^{2 n} \times S^{1}\right)$.

The reader is also referred to Ben Simon and Hartnick's work [BSH11, BSH12] regarding a general connection between quasi-morphisms and partial orders.

\subsubsection{Sandon-type metric}

In [San10] Sandon introduced an unbounded integer-valued conjugation-invariant norm on $\operatorname{Cont}_{0}^{c}\left(\mathbb{R}^{2 n} \times S^{1}\right)$, the identity component of the group of compactly supported contactomorphisms of $\mathbb{R}^{2 n} \times S^{1}$, and such norms have been further studied in AM13b, CS12, FPR12, Zap13. In what follows we will consider the norm $\nu$ defined in [FPR12, whose definition we will now recall.

Consider any orderable contact manifold $(V, \xi)$ for which there is a positive contact Hamiltonian $f>0$ such that $\widetilde{\phi}_{f}$ is in the center of $\widetilde{\operatorname{Cont}}_{0}(V)$. Examples of this are given by orderable contact manifolds with a periodic Reeb flow, for instance $\mathbb{R} P^{2 d-1}$ or any of the contact manifolds $\widehat{M}$ from Theorem 1.3 . The functionals on $\widehat{\operatorname{Cont}}_{0}(V)$

$$
\nu_{-}(\widetilde{\psi}):=\max \left\{n \in \mathbb{Z} \mid \widetilde{\phi}_{f}^{n} \preceq \widetilde{\psi}\right\} \quad \text { and } \quad \nu_{+}(\widetilde{\psi}):=\min \left\{n \in \mathbb{Z} \mid \widetilde{\psi} \preceq \widetilde{\phi}_{f}^{n}\right\}
$$

are conjugation-invariant, since $\widetilde{\phi}_{f}$ is in the center of $\widetilde{\operatorname{Cont}}_{0}(V)$, and

$$
\nu: \widetilde{\operatorname{Cont}}_{0}(V) \rightarrow \mathbb{Z} \quad \text { where } \nu(\widetilde{\phi}):=\max \left\{\left|\nu_{+}(\widetilde{\phi})\right|,\left|\nu_{-}(\widetilde{\phi})\right|\right\}
$$

defines a conjugation-invariant norm, by [FPR12, Theorem 2.4]. Using $\widetilde{\phi}_{f}$ is generated by a strictly positive contact Hamiltonian, it is easy to see from [EP00, Criterion 1.2.C] that $\nu\left(\widetilde{\phi}_{f}^{n}\right)=|n|$ for any $n \in \mathbb{Z}$ and hence $\nu$ is stably unbounded. This norm is related to monotone quasi-morphisms on $\widetilde{\operatorname{Cont}}_{0}(V)$ as follows:

Lemma 1.33. If $\mu: \widetilde{\operatorname{Cont}}_{0}(V) \rightarrow \mathbb{R}$ is a monotone quasi-morphism, then

$$
|\mu(\widetilde{\psi})| \leq \mu\left(\widetilde{\phi}_{f}\right) \nu(\widetilde{\psi}) .
$$

Note that $\mu\left(\widetilde{\phi}_{f}\right)>0$ since $\mu \neq 0$.

Proof. By the definition of $\nu_{ \pm}$and the fact that $\mu$ is monotone and homogeneous we have

$$
\nu_{-}(\widetilde{\psi}) \mu\left(\widetilde{\phi}_{f}\right) \leq \mu(\widetilde{\psi}) \leq \nu_{+}(\widetilde{\psi}) \mu\left(\widetilde{\phi}_{f}\right)
$$

from which the result follows. 
Next we show that the above norm is unbounded on subgroups of $\widetilde{\operatorname{Cont}}_{0}(V)$ associated to certain open subsets. For an open subset $U \subset V$ we let $\widetilde{\operatorname{Cont}_{0}}(U) \subset \widetilde{\operatorname{Cont}_{0}}(V)$ be the subgroup consisting of elements $\widetilde{\phi}_{h}$ where the Hamiltonian $h$ has compact support contained in $U$.

Theorem 1.34. If $U \subset V$ is an open subset containing a $\mu$-superheavy subset, then there is $\widetilde{\psi} \in{\widetilde{\operatorname{Cont}_{0}}}_{0}(U)$ with

$$
\lim _{n \rightarrow \infty} \frac{\nu\left(\widetilde{\psi}^{n}\right)}{n}>0
$$

that is $\nu$ is stably unbounded on $\widetilde{\operatorname{Cont}}_{0}(U)$.

Proof. By the above lemma we have

$$
\nu(\widetilde{\psi}) \geq \frac{|\mu(\widetilde{\psi})|}{\mu\left(\widetilde{\phi}_{f}\right)},
$$

therefore it suffices to produce an element $\widetilde{\psi} \in \widetilde{\operatorname{Cont}_{0}}(U)$ with $\mu(\widetilde{\psi}) \neq 0$. If $h$ is such that the restriction of $h$ to the superheavy subset is positive and $\operatorname{supp}(h) \subset U$, then since by definition $\mu\left(\widetilde{\phi}_{h}\right)>0$, we are done.

Colin-Sandon in CS12 used the notion of a discriminant point to define a non-degenerate bi-invariant metric on $\widetilde{\operatorname{Cont}}_{0}(V, \xi)$ for any contact manifold, which they called the discriminant metric. Using the relation between Givental's quasi-morphism $\mu_{\text {Giv }}$ with discriminant points, see Section 4.3.1 for more on this, Colin-Sandon were able to show the discriminant metric is stably unbounded on $\widetilde{\operatorname{Cont}}_{0}\left(\mathbb{R} P^{2 d-1}\right)$. It would be interesting to determine if the quasi-morphism $\mu$ : $\widetilde{\operatorname{Cont}}_{0}(\widehat{M}) \rightarrow \mathbb{R}$ we built in Theorem 1.3 can also be used to show the discriminant metric on $\widetilde{\operatorname{Cont}}_{0}(\widehat{M})$ is stably unbounded.

\subsection{Examples of even monotone polytopes}

Moment polytopes corresponding to closed monotone symplectic toric manifolds are known as smooth Fano polytopes. They have been classified by hand up to dimension 4 in Bat81, Bat99, Sat00, WW82 and there is an algorithm in Øbr07] for higher dimensions. We will now give various examples of even smooth Fano polytopes in $\mathbb{R}^{n}$ and their corresponding symplectic toric manifolds. For the polytopes we will just list the interior conormals $\left\{\nu_{j}\right\} \in \mathbb{Z}^{n}$ where $\left\{\epsilon_{1}, \ldots, \epsilon_{n}\right\}$ is the standard basis.

The first example is $\mathbb{C} P^{n}$ with conormals $\left\{\epsilon_{1}, \ldots, \epsilon_{n},-\left(\epsilon_{1}+\cdots+\epsilon_{n}\right)\right\}$ and in dimension two there are

$$
\mathbb{C} P^{2}, \mathbb{C} P^{1} \times \mathbb{C} P^{1}, \mathbb{C} P^{2} \# 3 \overline{\mathbb{C} P}^{2}
$$

where the last one has conormals $\left\{ \pm \epsilon_{1}, \pm \epsilon_{2}, \pm\left(\epsilon_{1}+\epsilon_{2}\right)\right\}$. In dimension three there are 18 smooth Fano polytopes by the classification [Bat81, WW82] and 8 are even. Four are basic

$$
\mathbb{C} P^{3}, \mathbb{C} P^{1} \times \mathbb{C} P^{2},\left(\mathbb{C} P^{1}\right)^{3}, \mathbb{C} P^{1} \times\left(\mathbb{C} P^{2} \# 3 \overline{\mathbb{C}} P^{2}\right)
$$

and the remaining four have the structure of toric bundles [MT10, Definition 3.10]: 

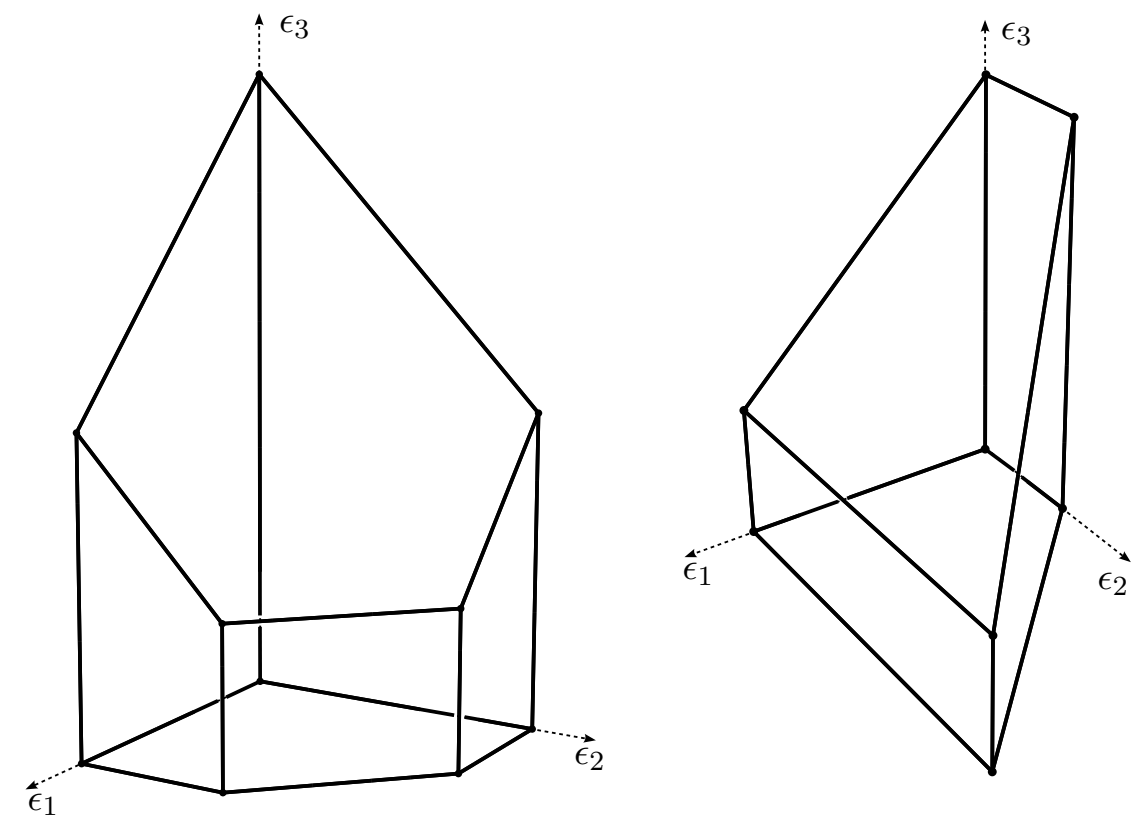

Figure 1: Left: The polytope for the $\left(\mathbb{C} P^{2} \# 2 \overline{\mathbb{C P}}^{2}\right)$-bundle over $\mathbb{C} P^{1}$ in (ii). Right: The polytope for the $\mathbb{C} P^{1}$-bundle $\mathbb{P}(\mathbb{C} \oplus \mathcal{O}(1,-1))$ over $\mathbb{C} P^{1} \times \mathbb{C} P^{1}$ in (iv).

(i) the $\mathbb{C} P^{1}$-bundle $\mathbb{P}(\mathbb{C} \oplus \mathcal{O}(2))$ over $\mathbb{C} P^{2}$ with conormals

$$
\left\{ \pm \epsilon_{1}, \epsilon_{2}, \epsilon_{3}, 2 \epsilon_{1}-\epsilon_{2}-\epsilon_{3}\right\}
$$

(ii) the $\left(\mathbb{C} P^{2} \# 2 \overline{\mathbb{C P}}^{2}\right.$ )-bundle $F_{3}^{4}$ (in the notation of [WW82]) over $\mathbb{C} P^{1}$ with conormals

$$
\left\{ \pm \epsilon_{1}, \pm \epsilon_{2},-\epsilon_{1}-\epsilon_{2}, \epsilon_{3},-\epsilon_{1}-\epsilon_{2}-\epsilon_{3}\right\}
$$

(iii) the $\mathbb{C} P^{1}$-bundle $\mathbb{P}(\mathbb{C} \oplus \mathcal{O}(1,1))$ over $\mathbb{C} P^{1} \times \mathbb{C} P^{1}$ with conormals

$$
\left\{ \pm \epsilon_{1}, \epsilon_{2}, \epsilon_{3}, \epsilon_{1}-\epsilon_{2}, \epsilon_{1}-\epsilon_{3}\right\}
$$

(iv) and the $\mathbb{C} P^{1}$-bundle $\mathbb{P}(\mathbb{C} \oplus \mathcal{O}(1,-1))$ over $\mathbb{C} P^{1} \times \mathbb{C} P^{1}$ with conormals

$$
\left\{ \pm \epsilon_{1}, \epsilon_{2}, \epsilon_{3}, \epsilon_{1}-\epsilon_{2},-\epsilon_{1}-\epsilon_{3}\right\}
$$

The example in (i) generalizes to the $\mathbb{C} P^{1}$-bundles $\mathbb{P}(\mathbb{C} \oplus \mathcal{O}(2 k))$ over $\mathbb{C} P^{n}$ where $0 \leq 2 k \leq n$. See Figure 1 for the polytopes from (ii) and (iv).

\section{Acknowledgements}

We are very grateful to Leonid Polterovich for his wonderful guidance and support with this paper. We thank Miguel Abreu, Alexander Givental, and Yael Karshon for helpful conversations and Milena Pabiniak, Sheila Sandon, and Egor Shelukhin for their comments on the manuscript. We also wish to thank the organizers and participants of the workshop 
"From Conservative Dynamics to Symplectic and Contact Topology" at the Lorentz Center (Leiden), the Geometry Seminar at Université Lyon 1 (Lyon), and the Geometry and Topology seminars at the University of Haifa and The Technion (Haifa), for the opportunity to present preliminary results of this work. Work on this paper began while visiting the Lorentz Center (Leiden) and our meetings at Tel Aviv University were instrumental in the completion of this project. The first named author thanks Leonid Polterovich and Yaron Ostrover for their warm hospitality during his stay at Tel Aviv University. Part of the writing was completed while the second named author was a postdoc at LMU (Munich), and he would like to acknowledge the nourishing research atmosphere and hospitality of this university. Finally we would like to thank the referee for their helpful comments, suggestions, and corrections.

\section{Proof of the main theorem}

In this section we will present the proof of Theorem 1.3. Section 2.1 contains the construction of the prequantization $\pi:(\widehat{M}, \alpha) \rightarrow(M, \omega)$ and Section 2.2 builds the announced quasi-morphisms $\mu$ : $\widetilde{\operatorname{Cont}}_{0}(\widehat{M}) \rightarrow \mathbb{R}$ using Theorem 1.8

\subsection{Constructing the family of contact manifolds}

The goal of this subsection is to present the construction of a prequantization $(\widehat{M}, \xi, \alpha)$ for an even closed monotone toric symplectic manifold $(M, \omega)$ with moment polytope

$$
\Delta=\left\{x \in \mathfrak{t}^{*} \mid\left\langle x, \nu_{j}\right\rangle+1 \geq 0 \text { for } j=1, \ldots, d\right\}
$$

as in (1.6) where $\nu_{j} \in \mathfrak{t}_{\mathbb{Z}}$ are primitive vectors and each one defines a different facet of the polytope $\Delta$. The polytope $\Delta$ is compact and smooth, meaning each $k$-codimensional face of $\Delta$ is the intersection of exactly $k$ facets and the $k$ associated conormals $\left\{\nu_{l_{1}}, \ldots, \nu_{l_{k}}\right\}$ can be extended to an integer basis for the lattice $\mathfrak{t}_{\mathbb{Z}}$. In (2.1) we have used the normalization $[\omega]=c_{1}(M)$ since $(M, \omega)$ is monotone and scaling the polytope $\Delta$ is equivalent to scaling $\omega$.

\subsubsection{The standard toric structure on $\mathbb{C}^{d}$ and Delzant's construction}

Let us briefly recall the standard toric structure on $\left(\mathbb{C}^{d}, \omega_{\text {std }}=d x \wedge d y\right)$. The action of $\mathbb{T}^{d}=\mathbb{R}^{d} / \mathbb{Z}^{d}$ on $\mathbb{C}^{d}$, which rotates each coordinate, is induced by the moment map

$$
P: \mathbb{C}^{d} \rightarrow \mathbb{R}^{d *} \quad \text { where } \quad\langle\lambda, P\rangle(z)=\pi \sum_{j=1}^{d} \lambda_{j}\left|z_{j}\right|^{2} \quad \text { for } \lambda=\left(\lambda_{1}, \ldots, \lambda_{d}\right) \in \mathbb{R}^{d} .
$$

Indeed for $\lambda \in \mathbb{R}^{d}$, the vector field

$$
X_{\lambda}(z)=2 \pi i\left(\lambda_{1} z_{1}, \ldots, \lambda_{d} z_{d}\right) \in \mathbb{C}^{d}=T_{z} \mathbb{C}^{d}
$$

is the Hamiltonian vector field for the function $\langle\lambda, P\rangle: \mathbb{C}^{d} \rightarrow \mathbb{R}$ and it gives the infinitesimal action of $\lambda$ on $\mathbb{C}^{d}$. Observe for the 1 -form

$$
\alpha_{\mathrm{std}}=\frac{1}{2} \sum_{j=1}^{d}\left(x_{j} d y_{j}-y_{j} d x_{j}\right)
$$


where $d \alpha_{\text {std }}=\omega_{\text {std }}$ one has

$$
\alpha_{\text {std }}\left(X_{\lambda}\right)=\langle\lambda, P\rangle \quad \text { and } \quad \iota_{X_{\lambda}} d \alpha_{\text {std }}=\iota_{X_{\lambda}} \omega_{\text {std }}=-d\langle\lambda, P\rangle .
$$

Delzant in [Del88] gave a way to reconstruct a closed symplectic toric manifold from its moment polytope using symplectic reduction of $\mathbb{C}^{d}$, which we will now recall in the case of the polytope $\Delta$ in 2.1 . Define the surjective linear map

$$
\beta_{\Delta}: \mathbb{R}^{d} \rightarrow \mathfrak{t} \quad \text { by } \quad \epsilon_{j} \mapsto \nu_{j} \quad \text { for } j=1, \ldots, d
$$

where $\left\{\epsilon_{j}\right\}_{j=1}^{d}$ are the standard basis vectors of $\mathbb{R}^{d}$ and $\nu_{j} \in \mathfrak{t}_{\mathbb{Z}}$ are conormals in 2.1). Since $\Delta$ is compact and smooth, we know $\beta_{\Delta}\left(\mathbb{Z}^{d}\right)=\mathfrak{t}_{\mathbb{Z}}$, and so we can define the connected subtorus

$$
\mathbb{K} \leq \mathbb{T}^{d} \text { to be the kernel of the induced map }\left[\beta_{\Delta}\right]: \mathbb{T}^{d} \rightarrow \mathbb{T}
$$

with Lie algebra

$$
\mathfrak{k}:=\operatorname{ker}\left(\beta_{\Delta}: \mathbb{R}^{d} \rightarrow \mathfrak{t}\right) .
$$

If $\iota^{*}: \mathbb{R}^{d *} \rightarrow \mathfrak{k}^{*}$ is dual to the inclusion $\mathfrak{k} \subset \mathbb{R}^{d}$, then the action of $\mathbb{K}$ on $\mathbb{C}^{d}$ has

$$
P_{\mathbb{K}}:=\iota^{*} \circ P: \mathbb{C}^{d} \rightarrow \mathfrak{k}^{*}
$$

for its moment map. The torus $\mathbb{K}$ acts freely on the regular level set

$$
P_{\mathbb{K}}^{-1}(c) \subset \mathbb{C}^{d} \text { where } \quad c:=\iota^{*}(1, \ldots, 1) \in \mathfrak{k}^{*}
$$

and for $\lambda \in \mathfrak{k}$ it follows from 2.4 that $\left.\left(\mathcal{L}_{X_{\lambda}} \omega_{\text {std }}\right)\right|_{P_{\mathbb{K}}^{-1}(c)}=0$. Therefore symplectic reduction gives a symplectic manifold $\left(M_{\Delta}, \omega_{\Delta}\right)$ where

$$
M_{\Delta}:=P_{\mathbb{K}}^{-1}(c) / \mathbb{K} \text { and the symplectic form } \omega_{\Delta} \text { is induced from }\left.\omega_{\text {std }}\right|_{P_{\mathbb{K}}^{-1}(c)} .
$$

It follows from Delzant's theorem [Del88] that $\left(M_{\Delta}, \omega_{\Delta}\right)$ and $(M, \omega)$ are equivariantly symplectomorphic as toric manifolds.

The following lemma that shows the significance of the assumption that $\Delta$ is an even moment polytope.

Lemma 2.1. Let $\tau \in \mathbb{T}^{d}$ be the element such that $\tau \cdot z=-z$ for $z \in \mathbb{C}^{d}$. The torus $\mathbb{K}$ from 2.5) contains the element $\tau$ if and only if $\Delta$ is even.

Proof. Note that $\tau=\left[\frac{1}{2}, \ldots, \frac{1}{2}\right]$ in $\mathbb{T}^{d}=\mathbb{R}^{d} / \mathbb{Z}^{d}$ and therefore since $\mathbb{T}=\mathfrak{t} / \mathfrak{t}_{\mathbb{Z}}$ it is clear from (2.5) that $\tau \in \mathbb{K}$ if and only if $\sum_{j=1}^{d} \frac{1}{2} \nu_{j} \in \mathfrak{t}_{\mathbb{Z}}$.

\subsubsection{The contact manifold $(\widehat{M}, \xi)$ from Delzant's construction of $(M, \omega)$}

Using Delzant's construction we will now describe the contact manifold $(\widehat{M}, \xi)$ associated to an even monotone symplectic toric manifold with moment polytope 2.1). Define

$$
\mathfrak{k}_{0}:=\operatorname{ker}(c: \mathfrak{k} \rightarrow \mathbb{R})
$$


to be the annihilator of the linear functional $c=\iota^{*}(1, \ldots, 1) \in \mathfrak{k}^{*}$ from $(2.7)$ and define

$$
\mathbb{K}_{0} \leq \mathbb{K} \text { to be the connected codimension } 1 \text { subtorus with Lie }\left(\mathbb{K}_{0}\right)=\mathfrak{k}_{0} .
$$

Since $\Delta$ in 2.1 is an even moment polytope, by Lemma 2.1 we know $\mathbb{K}_{0}+\langle\tau\rangle \leq \mathbb{K}$, where $\langle\tau\rangle \leq \mathbb{K}$ is the subgroup generated by $\tau$. Therefore $\mathbb{K}_{0}+\langle\tau\rangle$ also acts freely on the level set $P_{\mathbb{K}}^{-1}(c)$ from 2.7 .

The contact manifold $(\widehat{M}, \xi=\operatorname{ker} \alpha)$ is given by

$$
\widehat{M}:=P_{\mathbb{K}}^{-1}(c) /\left(\mathbb{K}_{0}+\langle\tau\rangle\right)
$$

and the contact form $\alpha$, which is induced from $\left.\alpha_{\text {std }}\right|_{P_{\mathbb{K}}^{-1}(c)}$, is well-defined because the infinitesimal action of $\mathbb{K}_{0}$ is tangent to ker $\alpha_{\text {std }}$ along $P_{\mathbb{K}}^{-1}(c)$, which follows from 2.4). For the circle $S^{1}=\mathbb{K} /\left(\mathbb{K}_{0}+\langle\tau\rangle\right)$, the natural projection map

$$
\pi:(\widehat{M}, \alpha) \rightarrow\left(M_{\Delta}, \omega_{\Delta}\right)
$$

defines a principal $S^{1}$-bundle and satisfies $\pi^{*} \omega_{\Delta}=d \alpha$ since $\omega_{\text {std }}=d \alpha_{\text {std }}$. Therefore by using a symplectomorphism $\left(M_{\Delta}, \omega_{\Delta}\right) \simeq(M, \omega)$, we have that $(2.11)$ is the desired prequantization in Theorem 1.3.

We will now present a formula for the period of the the Reeb vector field of $(\widehat{M}, \alpha)$ and hence the Euler class $e(\pi) \in H^{2}(M ; \mathbb{Z})$ of the principal $S^{1}$-bundle (2.11). For the functional $c: \mathfrak{k} \rightarrow \mathbb{R}$ from 2.7 , let

$$
c_{\mathfrak{k}} \in \mathbb{Z}
$$

be the positive generator of the image $c\left(\mathfrak{k}_{\mathbb{Z}}\right) \subset \mathbb{Z}$ of the integer lattice $\mathfrak{k}_{\mathbb{Z}}:=\mathfrak{k} \cap \mathbb{Z}^{d}$ and let

$$
\delta:=\left\{\begin{array}{ll}
1 & \text { if } \tau \in \mathbb{K}_{0} \\
2 & \text { if } \tau \notin \mathbb{K}_{0}
\end{array} .\right.
$$

Proposition 2.2. The Reeb vector field for $(\widehat{M}, \alpha)$ has period $\frac{c_{\mathfrak{k}}}{\delta}$ and the Euler class of $\pi:(\widehat{M}, \alpha) \rightarrow(M, \omega)$ equals

$$
e(\pi)=-\frac{\delta}{c_{\mathfrak{k}}} c_{1}(M) \in H^{2}(M ; \mathbb{Z}) .
$$

Proof. Recall for a principal $S^{1}$-bundle $\pi: V \rightarrow M$ that if $\alpha$ is a connection 1-form on $V$ and $\omega=\pi_{*}(d \alpha)$ is the curvature 2 -form on $M$, then the Euler class is given by [Mor01, Section $6.2(\mathrm{~d})]$

$$
e(\pi):=\frac{-1}{\int_{\pi^{-1}(m)} \alpha}[\omega] \in H^{2}(M ; \mathbb{Z}),
$$

the negative of the curvature form divided by the integral of the connection form over a fiber. For our prequantization $\int_{\pi^{-1}(m)} \alpha$ is the period of the Reeb vector field and we used the normalization $[\omega]=c_{1}(M)$, so it suffices to compute that $\int_{\pi^{-1}(m)} \alpha=\frac{c_{\mathfrak{k}}}{\delta}$.

Consider $\widehat{M}_{\Delta}:=P_{\mathbb{K}}^{-1}(c) / \mathbb{K}_{0}$ with 1 -form $\alpha_{\Delta}$ induced from $\alpha_{\text {std }}$. Under the identification $(M, \omega) \simeq\left(M_{\Delta}, \omega_{\Delta}\right)$ from 2.8 , the projection map defines a prequantization

$$
\pi_{\Delta}:\left(\widehat{M}_{\Delta}, \alpha_{\Delta}\right) \rightarrow(M, \omega)
$$


We will compute that $\int_{\pi_{\Delta}^{-1}(\bar{z})} \alpha_{\Delta}=c_{\mathfrak{k}}$ for any $\bar{z} \in M$, which will suffice since $\widehat{M}_{\Delta} \rightarrow \widehat{M}$ is a degree $\delta$ cover. By the definition of $\left(\widehat{M}_{\Delta}, \alpha_{\Delta}\right)$, its Reeb vector field can be represented by the infinitesimal action of $X_{\lambda}$ from 2.2 on $P_{\mathbb{K}}^{-1}(c)$ for any

$$
\lambda \in \mathfrak{k} \quad \text { such that }\langle\lambda, c\rangle=1 .
$$

For any such $\lambda$, the period of the Reeb orbit can also be characterized as the smallest $T>0$ so that $\exp (T \lambda) \in \mathbb{K}_{0}$ for the exponential map exp : $\mathfrak{k} \rightarrow \mathbb{K}$. Since $\left\langle\lambda_{0}, c\right\rangle=0$ for any $\lambda_{0} \in \mathfrak{k}_{0}$ and $\mathbb{K}_{0}$ is connected, we can choose $\lambda$ as in 2.13 so that the first return is at $\exp (T \lambda)=1 \in \mathbb{K}$. In this case $T \lambda \in \mathfrak{k}_{\mathbb{Z}}$ and $T=\langle T \lambda, c\rangle \in c\left(\mathfrak{k}_{\mathbb{Z}}\right)$, so therefore $T=c_{\mathfrak{k}}$ the minimal positive generator of $c\left(\mathfrak{k}_{\mathbb{Z}}\right)$.

Remark 2.3. Both options in 2.12) actually occur. For the case of $\mathbb{C} P^{n}$ we have $\tau \notin \mathbb{K}_{0}$, since $\mathbb{K}_{0}=1$, and for $\mathbb{C} P^{n} \times \mathbb{C} P^{n}$ below we do have $\tau \in \mathbb{K}_{0}$.

\subsubsection{An example in the case $M=\mathbb{C} P^{n-1} \times \mathbb{C} P^{n-1}$}

Consider the even toric monotone symplectic manifold

$$
(M, \omega)=\left(\mathbb{C} P^{n-1} \times \mathbb{C} P^{n-1}, n \sigma \oplus n \sigma\right)
$$

where $\int_{\mathbb{C} P^{1}} \sigma=1$. Its moment polytope is

$$
\left\{\left(x, x^{\prime}\right) \in\left(\mathbb{R}^{2 n-2}\right)^{*} \mid x_{j}+1 \geq 0,-\sum_{j=1}^{n-1} x_{j}+1 \geq 0, x_{j}^{\prime}+1 \geq 0,-\sum_{j=1}^{n-1} x_{j}^{\prime}+1 \geq 0\right\}
$$

where we have identified $\mathbb{T}=\mathbb{R}^{2 n-2} / \mathbb{Z}^{2 n-2}$. In this case $\mathbb{K}_{0} \leq \mathbb{K}$ are the subtori of $\mathbb{T}^{2 n}$ whose Lie algebras in $\mathbb{R}^{2 n}$ have bases

$$
\mathfrak{k}_{0}=\operatorname{span}\left\{\sum_{j=1}^{n} \epsilon_{j}-\sum_{j=1}^{n} \epsilon_{j}^{\prime}\right\} \quad \text { and } \quad \mathfrak{k}=\operatorname{span}\left\{\sum_{j=1}^{n} \epsilon_{j}, \sum_{j=1}^{n} \epsilon_{j}^{\prime}\right\} .
$$

The moment map $P_{\mathbb{K}}: \mathbb{C}^{2 n} \rightarrow \mathfrak{k}^{*}=\left(\mathbb{R}^{2}\right)^{*}$ for the action of $\mathbb{K}$ on $\mathbb{C}^{2 n}$ is

$$
P_{\mathbb{K}}\left(z, z^{\prime}\right)=\pi\left(\sum_{j=1}^{n}\left|z_{j}\right|^{2}, \sum_{j=1}^{n}\left|z_{j}^{\prime}\right|^{2}\right)
$$

and we have $P_{\mathbb{K}}^{-1}(c)$ is $S^{2 n-1} \times S^{2 n-1} \subset \mathbb{C}^{2 n}$ since

$$
P_{\mathbb{K}}^{-1}(c)=\left\{\left.\left(z, z^{\prime}\right) \in \mathbb{C}^{2 n}\left|\pi \sum_{j=1}^{n+1}\right| z_{j}\right|^{2}=\pi \sum_{j=1}^{n+1}\left|z_{j}^{\prime}\right|^{2}=n\right\} .
$$

The action of the circle $\mathbb{K}_{0}$ on $\mathbb{C}^{2 n}$ is given by $\zeta \cdot\left(z, z^{\prime}\right)=\left(\zeta z, \bar{\zeta} z^{\prime}\right)$ for $\zeta \in S^{1}$ the unit circle and note that $\tau \in \mathbb{K}_{0}$. The contact manifold is

$$
\widehat{M}=\left(S^{2 n-1} \times S^{2 n-1}\right) / \mathbb{K}_{0} \quad \text { with contact form } \alpha \text { induced by }\left.\left.\quad \alpha_{\text {std }}\right|_{S^{2 n-1}} \oplus \alpha_{\text {std }}\right|_{S^{2 n-1}} .
$$

The Reeb vector field $R_{\alpha}$ is represented by $X_{\lambda}$ with $\lambda=\frac{1}{2 n}(1, \ldots, 1) \in \mathbb{R}^{2 n}$ from 2.2 and it has period is $c_{\mathfrak{k}}=n$, so therefore the prequantization is the $\mathbb{R} / n \mathbb{Z}$-bundle

$$
\pi:(\widehat{M}, \alpha) \rightarrow\left(\mathbb{C} P^{n-1} \times \mathbb{C} P^{n-1}, n \sigma \oplus n \sigma\right) .
$$


Since the first Chern class $c_{1}\left(\mathbb{C} P^{n-1} \times \mathbb{C} P^{n-1}\right)=(n, n) \in H^{2}\left(\mathbb{C} P^{n-1} \times \mathbb{C} P^{n-1} ; \mathbb{Z}\right)$, we have

$$
e(\pi)=(-1,-1) \in H^{2}\left(\mathbb{C} P^{n-1} \times \mathbb{C} P^{n-1} ; \mathbb{Z}\right)
$$

from Proposition 2.2 .

Rescaling so that prequantization is a $\mathbb{R} / \mathbb{Z}$-bundle, we see that

$$
\pi: \widehat{M} \rightarrow\left(\mathbb{C} P^{n-1} \times \mathbb{C} P^{n-1}, \sigma \oplus \sigma\right)
$$

is the standard Boothby-Wang prequantization [BW58]. For the case of $n=2$, it is known that $\widehat{M}$ is contactomorphic to $U T^{*} S^{3}$ the unit cotangent bundle of $S^{3}$, for instance see [AM12, Section 6.1]. However when $n \geq 3$, it follows from [BW58, Theorem 8] that $\widehat{M}$ is not even topologically a unit cotangent bundle.

\subsection{Applying Theorem 1.8 to prove Theorem 1.3}

Proof of Theorem 1.3. Theorem 1.3 will be proved by applying Theorem 1.8 to Givental's quasi-morphism $\mu_{\text {Giv }}$ : $\widehat{\operatorname{Cont}}_{0}\left(\mathbb{R} P_{\gamma}^{2 d-1}\right) \rightarrow \mathbb{R}$ in the setting

$$
\left(\mathbb{R} P_{\gamma}^{2 d-1}, \xi_{\gamma}, \alpha_{\text {std }}\right) \supset\left(Y,\left.\alpha_{\text {std }}\right|_{Y}\right) \stackrel{\rho}{\longrightarrow}(\widehat{M}, \xi, \alpha)
$$

for an appropriate $\gamma$ and $Y$ that we describe below.

By [AM13a, Lemma 4.8] there is a primitive vector $\gamma=\left(\gamma_{1}, \ldots, \gamma_{d}\right) \in \mathfrak{k}_{\mathbb{Z}}:=\mathbb{Z}^{d} \cap \mathfrak{k}$ where each $\gamma_{j} \geq 1$. Fix such a $\gamma$ and consider the sphere $S_{\gamma}^{2 d-1}$ from (1.19). Note that

$$
P_{\mathbb{K}}^{-1}(c) \subset S_{\gamma}^{2 d-1}=\left\{z \in \mathbb{C}^{d} \mid\langle\gamma, P\rangle(z)=\langle\gamma, c\rangle\right\}
$$

since $z \in P_{\mathbb{K}}^{-1}(c)$ is equivalent to $\langle\lambda, P\rangle(z)=\langle\lambda, c\rangle$ for all $\lambda \in \mathfrak{k}$. Since $\Delta$ is even we know $\tau \in \mathbb{K}$ by Lemma 2.1 and modding out by the antipodal $\mathbb{Z}_{2}=\langle\tau\rangle$ action gives the submanifold

$$
Y:=P_{\mathbb{K}}^{-1}(c) /\langle\tau\rangle \subset\left(\mathbb{R} P_{\gamma}^{2 d-1}, \xi_{\gamma}\right)
$$

where recall $\left(\mathbb{R} P_{\gamma}^{2 d-1}, \xi_{\gamma}\right)=\left(S_{\gamma}^{2 d-1} /\langle\tau\rangle\right.$, ker $\left.\alpha_{\text {std }}\right)$. The natural projection map

$$
\rho: Y \rightarrow \widehat{M}
$$

is a principal $\left(\mathbb{K}_{0}+\langle\tau\rangle\right) /\langle\tau\rangle$-bundle and by the construction of the 1-form $\alpha$ from 2.10 it follows that $\rho^{*} \alpha=\left.\alpha_{\text {std }}\right|_{Y}$.

To verify the geometric setting 1.9$)$ of Theorem 1.8 it remains to prove $Y \subset \mathbb{R} P_{\gamma}^{2 d-1}$ is strictly coisotropic with respect to $\alpha_{\text {std }}$. Note that $P_{\mathbb{K}}^{-1}(c) \subset\left(\mathbb{C}^{d}, \omega_{\text {std }}\right)$ is a coisotropic submanifold, meaning for all $z \in P_{\mathbb{K}}^{-1}(c)$ :

$$
\left(T_{z} P_{\mathbb{K}}^{-1}(c)\right)^{\omega_{\text {std }}}:=\left\{X \in T_{z} \mathbb{C}^{d} \mid \iota_{X} \omega_{\text {std }}=0 \text { on } T_{z} P_{\mathbb{K}}^{-1}(c)\right\} \subset T_{z} P_{\mathbb{K}}^{-1}(c),
$$

since $P_{\mathbb{K}}^{-1}(c)$ is the regular level set of a moment map or as can be verified with (2.4). It now follows from 2.19) and $d \alpha_{\text {std }}=\omega_{\text {std }}$ that $P_{\mathbb{K}}^{-1}(c) \subset S_{\gamma}^{2 d-1}$ satisfies the condition (1.8) to be strictly coisotropic with respect to $\alpha_{\text {std }}$ and therefore so is $Y \subset \mathbb{R} P_{\gamma}^{2 d-1}$. 
Using the definition 2.7) of $P_{\mathbb{K}}^{-1}(c)$ we know

$$
\left\{\left|z_{1}\right|^{2}=\cdots=\left|z_{d}\right|^{2}=1 / \pi\right\} \subset P_{\mathbb{K}}^{-1}(c)
$$

since if $z \in\left\{\left|z_{1}\right|^{2}=\cdots=\left|z_{d}\right|^{2}=1 / \pi\right\}$, then for any $\lambda \in \mathfrak{k}$ one has

$$
\langle\lambda, P\rangle(z)=\pi \sum_{j=1}^{d} \lambda_{j} \frac{1}{\pi}=\sum_{j=1}^{d} \lambda_{j}=\langle\lambda, c\rangle
$$

where the last equality follows from the fact that $c:=\iota^{*}(1, \ldots, 1)$. Hence $T_{\mathbb{R} P} \subset Y$ for the torus $T_{\mathbb{R} P} \subset \mathbb{R} P_{\gamma}^{2 d-1}$ from 1.24 , which is $\mu_{\text {Giv }}$-superheavy by Lemma 1.22 . Therefore by Theorem 1.11 we know $Y \subset \mathbb{R} P_{\gamma}^{2 d-1}$ is $\mu_{\text {Giv }}$-subheavy. Applying Theorem 1.8 to 2.16

constructs the desired monotone quasi-morphism $\bar{\mu}$ : $\widetilde{\operatorname{Cont}}_{0}(\widehat{M}, \xi) \rightarrow \mathbb{R}$ with the vanishing property.

Remark 2.4. For any closed even symplectic toric manifold $(M, \omega, \mathbb{T})$ the construction in this section can be modified to produce a prequantization $\pi:(\widehat{M}, \alpha) \rightarrow(M, \omega)$ that is constructed by contact reduction of a real projective space. Without the monotonicity assumption however, one needs to replace $c=\iota^{*}(1, \ldots, 1)$ with $\iota^{*}\left(a_{1}, \ldots, a_{d}\right)$ where the $a_{j}$ are the support constants in the moment polytope (1.6). With this change 2.20 no longer holds so the reduction will not pass through the superheavy torus $T_{\mathbb{R} P}$. This is similar to the proof of [AM13a, Proposition 4.9].

\section{Proof of the reduction theorem for quasi-morphisms}

In this section we will present the proof of Theorem 1.8 .

\subsection{Preliminary lemmas}

\subsubsection{Geometric setting of Theorem 1.8}

Let us begin by collecting a few lemmas about the geometric setting of Theorem 1.8 .

$$
(V, \xi, \alpha) \supset\left(Y,\left.\alpha\right|_{Y}\right) \stackrel{\rho}{\longrightarrow}(\bar{V}, \bar{\xi}, \bar{\alpha})
$$

where $(V, \xi, \alpha)$ and $(\bar{V}, \bar{\xi}, \bar{\alpha})$ are closed contact manifolds, $Y \subset V$ is a closed submanifold that is strictly coisotropic with respect to $\alpha$, and $\rho$ is a fiber bundle such that $\rho^{*} \bar{\alpha}=\left.\alpha\right|_{Y}$.

Lemma 3.1. The map $d \rho: T Y \rightarrow T \bar{V}$ relates the Reeb vector fields: $\left.d \rho \circ R_{\alpha}\right|_{Y}=R_{\bar{\alpha}} \circ \rho$.

Proof. Note that by Definition 1.6 of strictly coisotropic the Reeb vector field $\left.R_{\alpha}\right|_{Y}$ is tangent to $Y$. To show $d \rho \circ R_{\alpha}=R_{\bar{\alpha}} \circ \rho$ one computes

$$
\bar{\alpha}\left(d \rho\left(R_{\alpha}\right)\right)=\rho^{*} \bar{\alpha}\left(R_{\alpha}\right)=\alpha\left(R_{\alpha}\right)=1
$$

and for any $u \in T Y$ one has

$$
d \bar{\alpha}\left(d \rho\left(R_{\alpha}\right), d \rho(u)\right)=d\left(\rho^{*} \bar{\alpha}\right)\left(R_{\alpha}, u\right)=d \alpha\left(R_{\alpha}, u\right)=0
$$

which proves $\iota_{d \rho\left(R_{\alpha}\right)} d \bar{\alpha}=0$ since $d \rho: T Y \rightarrow T \bar{V}$ is surjective. By definition of $R_{\bar{\alpha}}$ this proves $\left.d \rho \circ R_{\alpha}\right|_{Y}=R_{\bar{\alpha}} \circ \rho$. 
For an $\bar{h} \in C^{\infty}([0,1] \times \bar{V})$, an extension of $\bar{h}$ will be any $h \in C^{\infty}([0,1] \times V)$ such that

$$
\left.h\right|_{[0,1] \times Y}=\rho^{*} \bar{h} .
$$

Lemma 3.2. If $h \in C^{\infty}([0,1] \times V)$ is an extension of $\bar{h} \in C^{\infty}([0,1] \times \bar{V})$, then

(i) the contact vector field $\left.X_{h_{t}}\right|_{Y}$ is tangent to $Y$,

(ii) the contact vector fields of $h, \bar{h}$ are related by $d \rho:\left.d \rho \circ X_{h_{t}}\right|_{Y}=X_{\bar{h}_{t}} \circ \rho$, and

(iii) as maps $\left.\rho \circ \phi_{h}^{t}\right|_{Y}=\phi_{h}^{t} \circ \rho: Y \rightarrow \bar{V}$ and $\left.\rho \circ\left(\phi_{h}^{t}\right)^{-1}\right|_{Y}=\left(\phi_{\bar{h}}^{t}\right)^{-1} \circ \rho: Y \rightarrow \bar{V}$ for all $t \in[0,1]$.

Proof. It suffices to prove (i) for autonomous $h \in C^{\infty}(V)$ and $\bar{h} \in C^{\infty}(\bar{V})$. Let $u \in T Y$, then by the definition of $X_{h}$ from $(1.2)$ and the relations

$$
\rho^{*} \bar{\alpha}=\left.\alpha\right|_{Y}, \quad \rho^{*} \bar{h}=\left.h\right|_{Y},\left.\quad d \rho \circ R_{\alpha}\right|_{Y}=R_{\bar{\alpha}} \circ \rho
$$

we have

$$
d \alpha\left(X_{h}, u\right)=-d h(u)+d h\left(R_{\alpha}\right) \alpha(u)=-d \bar{h}(d \rho(u))+d \bar{h}\left(R_{\bar{\alpha}}\right) \bar{\alpha}(d \rho(u))=d \bar{\alpha}\left(X_{\bar{h}}, d \rho(u)\right) .
$$

Since $X_{\bar{h}}=d \rho(v)$ for some $v \in T Y$, taking any $u \in(T Y)^{d \alpha} \subset T Y$ (see (1.8)) we get

$$
d \alpha\left(X_{h}, u\right)=d \bar{\alpha}(d \rho(v), d \rho(u))=d \alpha(v, u)=0,
$$

and hence $\left.X_{h}\right|_{Y} \in\left((T Y)^{d \alpha}\right)^{d \alpha}$. Since $Y$ is strictly coisotropic, it follows from $(1.8)$ that $\left((T Y)^{d \alpha}\right)^{d \alpha}=T Y$ and hence $\left.X_{h}\right|_{Y} \in T Y$.

For item (ii), similar considerations as above show

$$
\bar{\alpha}\left(d \rho\left(X_{h}\right)\right)=\alpha\left(X_{h}\right)=\bar{h} \quad \text { at the point } \rho(y)
$$

and likewise for any $u \in T Y$

$$
d \bar{\alpha}\left(d \rho\left(X_{h}\right), d \rho(u)\right)=d \alpha\left(X_{h}, u\right)=-d h(u)+d h\left(R_{\alpha}\right) \alpha(u)=-d \bar{h}(d \rho(u))+d \bar{h}\left(R_{\bar{\alpha}}\right) \bar{\alpha}(d \rho(u)) .
$$

Since $d \rho: T Y \rightarrow T \bar{V}$ is surjective this shows $\left.d \rho \circ X_{h}\right|_{Y}=X_{\bar{h}} \circ \rho$.

The first part of item (iii) immediately follows from item (ii). The second part of item (iii) follows from the first part via $\left.\rho \circ\left(\phi_{h}^{t}\right)^{-1}\right|_{Y}=\left.\left(\phi_{\frac{t}{h}}\right)^{-1} \circ \phi \frac{t}{h} \circ \rho \circ\left(\phi_{h}^{t}\right)^{-1}\right|_{Y}=\left(\phi \frac{t}{h}\right)^{-1} \circ \rho$.

\subsubsection{Using the subheavy assumption}

Recall that in Theorem 1.8 that in addition to the geometric setting in (3.1), $Y \subset V$ is subheavy with respect to a monotone quasi-morphism $\mu$ : $\widetilde{\operatorname{Cont}}_{0}(V) \rightarrow \mathbb{R}$.

Lemma 3.3. If $h \in C^{\infty}([0,1] \times V)$ is such that $\left.X_{h_{t}}\right|_{Y} \in T Y$ for all $t \in[0,1]$ and

$$
\left.\rho \circ \phi_{h}^{t}\right|_{Y}=\rho: Y \rightarrow \bar{V} \quad \text { for all } t \in[0,1],
$$

then $\mu\left(\widetilde{\phi}_{h}\right)=0$. 
Proof. Differentiating 3.2 with respect to $t$ gives $d \rho\left(X_{h_{t}}\right)=0$ and hence on $Y$ :

$$
h_{t}=\alpha\left(X_{h_{t}}\right)=\bar{\alpha}\left(d \rho\left(X_{h_{t}}\right)\right)=0 .
$$

Now pick autonomous Hamiltonians $g, k \in C^{\infty}(V)$ so that $g \leq h \leq k$ and $\left.g\right|_{Y}=\left.k\right|_{Y}=0$. Since $Y$ is $\mu$-subheavy it follows that $\mu\left(\widetilde{\phi}_{g}\right)=\mu\left(\widetilde{\phi}_{k}\right)=0$ and therefore $\mu\left(\widetilde{\phi}_{h}\right)=0$ since $\mu$ is monotone.

Lemma 3.4. Let $\bar{h} \in C^{\infty}([0,1] \times \bar{V})$ be such that $\left\{\phi_{\bar{h}}^{t}\right\}_{t \in[0,1]}$ is a contractible loop in Cont $_{0}(\bar{V})$. For any contact Hamiltonian $h \in C^{\infty}([0,1] \times V)$ that is an extension of $\bar{h}$ it follows $\mu\left(\widetilde{\phi}_{h}\right)=0$.

Proof. Let $\Phi:[0,1]^{2} \rightarrow \operatorname{Cont}_{0}(\bar{V})$ be a null-homotopy of loops for $\left\{\phi_{\bar{h}}^{t}\right\}$, so $\left\{\Phi_{t}^{s}\right\}_{t \in[0,1]}$ is a loop of contactomorphisms of $\bar{V}$ for fixed $s$ where $\Phi_{t}^{0}=\mathrm{id}_{\bar{V}}$ and $\Phi_{t}^{1}=\phi_{\bar{h}}^{t}$. Let

$$
\bar{H}^{s}:[0,1] \times \bar{V} \rightarrow \mathbb{R}
$$

be the contact Hamiltonian generating the contact isotopy $\left\{\Phi_{t}^{s}\right\}_{t \in[0,1]}$ for fixed $s$ and note $\bar{H}_{t}^{1}=\bar{h}_{t}$.

Let $H:[0,1]^{2} \times V \rightarrow \mathbb{R}$ be an extension of $\bar{H}$ so that $\left.H_{t}^{s}\right|_{Y}=\bar{H}_{t}^{s} \circ \rho$ and $H_{t}^{1}=h_{t}$ is the chosen extension of $\bar{h}$. Let $\left\{\Psi_{t}^{s}\right\}_{t \in[0,1]}$ be the contact isotopy of $V$ generated by

$$
H_{.}^{s}:[0,1] \times V \rightarrow \mathbb{R}
$$

for fixed $s$. It follows from Lemma 3.2 that $\left.\rho \circ \Psi_{t}^{s}\right|_{Y}=\Phi_{t}^{s} \circ \rho: Y \rightarrow \bar{V}$ for all $s$ and $t$. In particular the concatenation of paths

$$
\left\{\Psi_{0}^{1-u}\right\}_{u \in[0,1]} \#\left\{\Psi_{u}^{0}\right\}_{u \in[0,1]} \#\left\{\Psi_{1}^{u}\right\}_{u \in[0,1]}
$$

defines an isotopy $\left\{\psi^{u}\right\}_{u \in[0,1]}$ in $\operatorname{Cont}_{0}(V)$ such that $\psi^{u}(Y)=Y$ and $\left.\rho \circ \psi^{u}\right|_{Y}=\mathrm{id}_{\bar{V}} \circ \rho$ since

$$
\operatorname{id}_{\bar{V}}=\left\{\Phi_{0}^{1-u}\right\}_{u \in[0,1]} \#\left\{\Phi_{u}^{0}\right\}_{u \in[0,1]} \#\left\{\Phi_{1}^{u}\right\}_{u \in[0,1]} \quad \text { in } \operatorname{Cont}_{0}(\bar{V}) .
$$

Let $\widetilde{\psi} \in \widetilde{\operatorname{Cont}}_{0}(V)$ be the element represented by $\left\{\psi^{u}\right\}_{u \in[0,1]}$. By Lemma 3.3 we know $\mu(\widetilde{\psi})=0$ and hence $\mu\left(\widetilde{\phi}_{h}\right)=\mu(\widetilde{\psi})=0$ since $\left\{\psi^{u}\right\}_{u \in[0,1]}$ is homotopic with fixed endpoints to the isotopy $\left\{\Psi_{t}^{1}\right\}_{t \in[0,1]}=\left\{\phi_{h}^{t}\right\}_{t \in[0,1]}$.

\subsection{Proof of Theorem 1.8}

Let $\mathcal{P} \operatorname{Cont}_{0}(\bar{V})$ denote the group of contact isotopies of $(\bar{V}, \bar{\xi})$ based at the identity with time-wise composition as the product: $\left\{\phi_{\frac{t}{h}}\right\} *\left\{\phi_{\frac{t}{k}}\right\}=\left\{\phi_{\frac{t}{h}}^{t} \circ \phi_{\frac{t}{k}}\right\}$. Passing to homotopy classes of isotopies with fixed endpoints is a group homomorphism $\mathcal{P} \operatorname{Cont}_{0}(\bar{V}) \rightarrow \widetilde{\operatorname{Cont}_{0}}(\bar{V})$ whose kernel consists of contractible loops based at the identity.

Proof of Theorem 1.8. We will break the proof of Theorem 1.8 into a few steps.

Independence of choice of extension: We will first prove

$$
\bar{\mu}: \mathcal{P} \operatorname{Cont}_{0}(\bar{V}) \rightarrow \mathbb{R} \quad \text { defined by } \quad \bar{\mu}\left(\left\{\phi_{\bar{h}}^{t}\right\}\right)=\mu\left(\widetilde{\phi}_{h}\right)
$$


where $h \in C^{\infty}([0,1] \times V)$ is any extension of $\bar{h} \in C^{\infty}([0,1] \times \bar{V})$ is well-defined. So let $h$ and $k$ both be extensions of $\bar{h}$. For any positive integer $m$ by Lemma 3.2(iii) it follows

$$
\left.\rho \circ\left(\phi_{h}^{t}\right)^{m} \circ\left(\phi_{k}^{t}\right)^{-m}\right|_{Y}=\left(\phi_{\bar{h}}^{t}\right)^{m} \circ\left(\phi_{\bar{k}}^{t}\right)^{-m} \circ \rho=\rho
$$

and hence $\mu\left(\widetilde{\phi}_{h}^{m} \widetilde{\phi}_{k}^{-m}\right)=0$ by Lemma 3.3 . Using that $\mu$ is a homogeneous quasi-morphism (1.1) we have

$$
\left|\mu\left(\widetilde{\phi}_{h}\right)-\mu\left(\widetilde{\phi}_{k}\right)\right|=\frac{1}{m}\left|\mu\left(\widetilde{\phi}_{h}^{m}\right)-\mu\left(\widetilde{\phi}_{k}^{m}\right)\right| \leq \frac{1}{m}\left(D+\mu\left(\widetilde{\phi}_{h}^{m} \widetilde{\phi}_{k}^{-m}\right)\right)=\frac{D}{m}
$$

and taking the limit as $m \rightarrow \infty$ shows $\mu\left(\widetilde{\phi}_{h}\right)=\mu\left(\widetilde{\phi}_{k}\right)$.

Homogeneous quasi-morphism on $\mathcal{P C o n t}_{0}(\bar{V})$ : We will first show that (3.3) defines a quasi-morphism. Let $g, h, k \in C^{\infty}([0,1] \times V)$ be extensions of $\bar{g}, \bar{h}, \bar{k} \in C^{\infty}([0,1] \times \bar{V})$ where $\bar{g}$ generates the product of $\bar{h}$ and $\bar{k}$, that is $\phi_{\bar{g}}^{t}=\phi_{\bar{h}}^{t} \circ \phi_{\bar{k}}^{t}$. By Lemma 3.2 (iii) we have

$$
\left.\rho \circ\left(\phi_{g}^{t}\right)^{-1} \circ \phi_{h}^{t} \circ \phi_{k}^{t}\right|_{Y}=\left(\phi_{\bar{g}}^{t}\right)^{-1} \circ \phi_{\bar{h}}^{t} \circ \phi_{\bar{k}}^{t} \circ \rho=\rho
$$

so by Lemma 3.3 it follows $\mu\left(\widetilde{\phi}_{g}^{-1} \widetilde{\phi}_{h} \widetilde{\phi}_{k}\right)=0$. If $D$ is as in 1.1 for $\mu$, it follows

$$
\left|\bar{\mu}\left(\left\{\phi_{\bar{h}}^{t}\right\} *\left\{\phi_{\bar{k}}^{t}\right\}\right)-\bar{\mu}\left(\left\{\phi_{\bar{h}}^{t}\right\}\right)-\bar{\mu}\left(\left\{\phi_{\bar{k}}^{t}\right\}\right)\right|=\left|\mu\left(\widetilde{\phi}_{g}\right)-\mu\left(\widetilde{\phi}_{h}\right)-\mu\left(\widetilde{\phi}_{k}\right)\right| \leq 2 D
$$

and therefore $\bar{\mu}$ in 3.3 is a quasi-morphism.

We will now show that $\bar{\mu}$ is homogeneous. If $h, g^{(m)} \in C^{\infty}([0,1] \times V)$ are extensions of $\bar{h}, \bar{g} \in C^{\infty}([0,1] \times \bar{V})$ where $\phi_{\bar{g}}^{t}=\left(\phi_{\bar{h}}^{t}\right)^{m}$ for an integer $m \in \mathbb{Z}$, then again Lemma 3.3 shows $\mu\left(\widetilde{\phi}_{g^{(m)}}^{-1} \widetilde{\phi}_{h}^{m}\right)=0$. It follows

$$
\left|\bar{\mu}\left(\left\{\phi_{\bar{h}}^{t}\right\}^{m}\right)-m \bar{\mu}\left(\left\{\phi_{h}^{t}\right\}\right)\right|=\left|\mu\left(\widetilde{\phi}_{g^{(m)}}\right)-\mu\left(\widetilde{\phi}_{h}^{m}\right)\right| \leq D+\left|\mu\left(\widetilde{\phi}_{g^{(m)}}^{-1} \widetilde{\phi}_{h}^{m}\right)\right|=D .
$$

Dividing (3.4) by $m$ and taking $m \rightarrow \infty$ shows that

$$
\lim _{m \rightarrow \infty} \frac{\bar{\mu}\left(\left\{\phi_{\bar{h}}^{t}\right\}^{m}\right)}{m}=\bar{\mu}\left(\left\{\phi_{\bar{h}}^{t}\right\}\right)
$$

and it follows from (3.5) that $\bar{\mu}$ is homogeneous, see for instance [Cal09, Lemma 2.21].

Descent to a quasi-morphism on $\widetilde{\operatorname{Cont}}_{0}(\bar{V})$ : Since $\bar{\mu}: \mathcal{P} \operatorname{Cont}_{0}(\bar{V}) \rightarrow \mathbb{R}$ vanishes on the kernel of the map $\mathcal{P} \operatorname{Cont}_{0}(\bar{V}) \rightarrow \widetilde{\operatorname{Cont}_{0}}(\bar{V})$ by Lemma 3.4 it follows that $\bar{\mu}$ in (3.3) descends

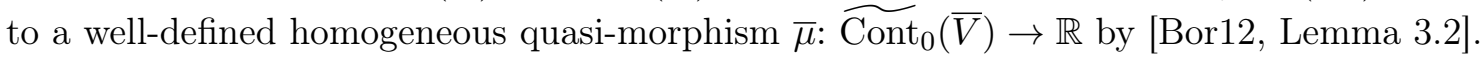

Non-zero: To see the quasi-morphism $\bar{\mu}: \widetilde{\operatorname{Cont}_{0}}(\bar{V}) \rightarrow \mathbb{R}$ is not zero, let $\bar{h} \in C^{\infty}(\bar{V})$ be any positive contact Hamiltonian and pick $h \in C^{\infty}(V)$ to be a positive extension. Since $V$ is $\mu$-superheavy by Proposition 1.10 (iv) it follows that $\bar{\mu}\left(\widetilde{\phi}_{\bar{h}}\right)=\mu\left(\widetilde{\phi}_{h}\right)>0$.

Monotone: If $\bar{h} \leq \bar{k}$, then one can pick extensions $h$ and $k$ such that $h \leq k$. Since $\mu$ is monotone it follows $\mu\left(\widetilde{\phi}_{h}\right) \leq \mu\left(\widetilde{\phi}_{k}\right)$ and therefore $\bar{\mu}\left(\widetilde{\phi}_{\bar{h}}\right) \leq \bar{\mu}\left(\widetilde{\phi}_{\bar{k}}\right)$ by definition.

Vanishing: Assume now that $\mu$ has the vanishing property. Let $U \subset \bar{V}$ be an open set that is displaceable by an element of $\operatorname{Cont}_{0}(\bar{V})$, then it follows from Lemma 3.2 (iii) that an open neighborhood $N \subset V$ of $\rho^{-1}(U) \subset Y$ is displaceable by an element of $\operatorname{Cont}_{0}(V)$. Now if $\bar{h} \in C^{\infty}([0,1] \times \bar{V})$ has $\operatorname{supp}(\bar{h}) \subset[0,1] \times U$, then there is an extension $h \in C^{\infty}([0,1] \times V)$ of 
$\bar{h}$ with $\operatorname{supp}(h) \subset[0,1] \times N$. Since $\mu$ has the vanishing property it follows $\mu\left(\widetilde{\phi}_{h}\right)=0$ and so by definition $\bar{\mu}\left(\widetilde{\phi}_{\bar{h}}\right)=0$ as well. Therefore $\bar{\mu}$ also has the vanishing property.

$C^{0}$-continuity: Assume that $\mu$ is $C^{0}$-continuous and let $\bar{h}^{(n)} \in C^{\infty}([0,1] \times \bar{V})$ be a sequence of contact Hamiltonians $C^{0}$-converging to $\bar{h} \in C^{\infty}([0,1] \times \bar{V})$. Then we can pick extensions $h^{(n)}$ and $h$ in $C^{\infty}([0,1] \times V)$ with $C^{0}$-convergence $h^{(n)} \rightarrow h$. Since $\mu$ is $C^{0}$-continuous, we have $\mu\left(\widetilde{\phi}_{h^{(n)}}\right) \rightarrow \mu\left(\widetilde{\phi}_{h}\right)$ and hence $\bar{\mu}\left(\widetilde{\phi}_{\bar{h}^{(n)}}\right) \rightarrow \bar{\mu}\left(\widetilde{\phi}_{h}\right)$ as well. Therefore $\bar{\mu}$ is $C^{0}$-continuous.

\section{Proof of rigidity and vanishing results}

In this section we will present the remaining proofs.

\subsection{Proof of rigidity results from Section 1.2}

We will first prove the following lemma that shows that there is no difference between positive and negative in terms of defining a subset to be superheavy with respect to a quasimorphism on $\widehat{\operatorname{Cont}}_{0}(V)$.

Lemma 4.1. If $\mu$ : $\widetilde{\mathrm{Cont}_{0}}(V, \xi) \rightarrow \mathbb{R}$ is a monotone quasi-morphism and $Y \subset V$ is a closed subset, then $Y$ is $\mu$-superheavy if and only if $\mu\left(\widetilde{\phi}_{h}\right)<0$ for all autonomous contact Hamiltonians where $\left.h\right|_{Y}<0$.

Proof. If $h$ is autonomous, then $\widetilde{\phi}_{h}^{-1}$ is generated by the contact Hamiltonian $-h$ and therefore $\mu\left(\widetilde{\phi}_{-h}\right)=-\mu\left(\widetilde{\phi}_{h}\right)$ since $\mu$ is homogeneous. The lemma now follows from the definition of $Y$ being superheavy from Definition 1.9 .

Let us now prove Proposition 1.10 and Theorem 1.11, detailing the basic properties of superheavy and subheavy sets.

Proof of Proposition 1.10. To prove item (i) recall that any two contact forms $\alpha$ and $\alpha^{\prime}$ for $\xi$ differ by multiplication by a positive function $f: V \rightarrow \mathbb{R}: \alpha^{\prime}=f \alpha$. If $h \in C^{\infty}([0,1] \times V)$ is the contact Hamiltonian associated to the contact isotopy $\left\{\phi^{t}\right\}_{t \in[0,1]}$ using the form $\alpha$, then $f \cdot h$ is the contact Hamiltonian associated to the same isotopy using the form $\alpha^{\prime}$. Hence $\left.h\right|_{[0,1] \times Y}>0$ if and only if $\left.f \cdot h\right|_{[0,1] \times Y}>0$, so $\mu$-superheaviness is independent of contact form and likewise for $\mu$-subheaviness.

Item (ii) is immediate since if $\left.h\right|_{Y}>0$, then $\left.h\right|_{Z}>0$ and hence $\mu\left(\widetilde{\phi}_{h}\right)>0$ since $Z$ is $\mu$-superheavy. The argument for $\mu$-subheaviness is analogous.

To prove (iii), first note homogeneous quasi-morphisms are conjugation-invariant so

$$
\mu\left(\widetilde{\phi}_{h}\right)=\mu\left(\psi^{-1} \widetilde{\phi}_{h} \psi\right)
$$

for any $h \in C^{\infty}(V)$ and $\psi \in \operatorname{Cont}_{0}(V)$, where we use the natural action of $\operatorname{Cont}_{0}(V)$ on $\widetilde{\operatorname{Cont}}_{0}(V)$ by conjugation. Furthermore $\psi^{-1} \widetilde{\phi}_{h} \psi=\widetilde{\phi}_{g}$ where

$$
g:=\alpha\left(d \psi^{-1}\left(X_{h}\right) \circ \psi\right)=(f \cdot h) \circ \psi
$$

for some positive function $f \in C^{\infty}(V)$, so in particular $\left.g\right|_{Y}>0$ if and only if $\left.h\right|_{\psi(Y)}>0$. Hence $Y$ is $\mu$-superheavy if and only if $\psi(Y)$ is $\mu$-superheavy and likewise for $\mu$-subheavy. 
For item (iv) recall that we assume all quasi-morphisms are homogeneous and non-zero. Suppose there is an $h \in C^{\infty}(V)$ such that $h>0$ and $\mu\left(\widetilde{\phi}_{h}\right)=0$. For any integer $m$ it follows that $\mu\left(\widetilde{\phi}_{m h}\right)=\mu\left(\widetilde{\phi}_{h}^{m}\right)=0$ since $h$ is autonomous. Since for any $k \in C^{\infty}([0,1] \times V)$ there is a positive integer $m$ such that $-m h \leq k \leq m h$, it follows from the monotonicity of $\mu$ that $\mu\left(\widetilde{\phi}_{k}\right)=0$. Therefore $\mu=0$, which is a contradiction.

Proof of Theorem 1.11. For item (i), let $Y$ be $\mu$-superheavy and $h$ be an autonomous contact Hamiltonian where $\left.h\right|_{Y}=0$. Recall for any $\phi \in \operatorname{Cont}_{0}(V)$ that $\phi^{*} \alpha=k \alpha$ where $k: V \rightarrow \mathbb{R}$ is a positive function. It follows then for any positive integer $m$ and real number $\epsilon>0$ that

$$
g_{t}:=\alpha\left(X_{m h}+d \phi_{m h}^{t}\left(m \epsilon R_{\alpha}\right) \circ\left(\phi_{m h}^{t}\right)^{-1}\right)
$$

which is the contact Hamiltonian so that $\phi_{g}^{t}=\phi_{m h}^{t} \phi_{m \epsilon}^{t}$ for all $t \in[0,1]$, satisfies $\left.g_{t}\right|_{Y}>\delta$ for some $\delta>0$. Using that $Y$ is $\mu$-superheavy and $\mu$ is monotone we have

$$
\mu\left(\widetilde{\phi}_{m h} \widetilde{\phi}_{m \epsilon}\right)=\mu\left(\widetilde{\phi}_{g}\right)>0
$$

Since $h$ is autonomous, $\widetilde{\phi}_{m h}=\widetilde{\phi}_{h}^{m}$, and using $\mu$ is a homogeneous quasi-morphism (1.1) we get

$$
m \mu\left(\widetilde{\phi}_{h}\right)=\mu\left(\widetilde{\phi}_{m h}\right) \geq \mu\left(\widetilde{\phi}_{m h} \widetilde{\phi}_{m \epsilon}\right)+\mu\left(\widetilde{\phi}_{m \epsilon}^{-1}\right)-D>\mu\left(\widetilde{\phi}_{m \epsilon}^{-1}\right)-D=-m \epsilon \mu\left(\widetilde{\phi}_{1}\right)-D .
$$

By dividing through by $m$ and taking the limit as $m \rightarrow \infty$ gives $\mu\left(\widetilde{\phi}_{h}\right)>-\epsilon \mu\left(\widetilde{\phi}_{1}\right)$ for all $\epsilon>0$, and therefore taking $\epsilon \rightarrow 0$ gives

$$
\mu\left(\widetilde{\phi}_{h}\right) \geq 0 .
$$

One proves $\mu\left(\widetilde{\phi}_{h}\right) \leq 0$ similarly using Lemma 4.1 . Therefore $\mu\left(\widetilde{\phi}_{h}\right)=0$ and hence $Y$ is $\mu$-subheavy.

To prove item (ii), suppose that $Y$ and $Z$ are disjoint and pick a contact Hamiltonian $h$ so that $\left.h\right|_{Y}>0$ and $\left.h\right|_{Z}=0$, which is possible since $Y, Z$ are closed subsets. This leads to a contradiction since by the definitions this implies $\mu\left(\phi_{h}\right)>0$ and $\mu\left(\phi_{h}\right)=0$.

Next up is the proof of Theorem 1.17 about the existence of nondisplaceable pre-Lagrangians in prequantizations of toric symplectic manifolds.

Proof of Theorem 1.17. Let $P: M^{2 n} \rightarrow \Delta \subset \mathbb{R}^{n}$ be a moment map for the toric structure on $M$, let $\pi:(V, \alpha) \rightarrow(M, \omega)$ be the prequantization map, and let $\widehat{P}=P \circ \pi: V \rightarrow \Delta$. Every fiber of $\widehat{P}$ is either a pre-Lagrangian torus or a sits over a strictly isotropic torus in $M$ and the latter are always displaceable [Lau86], so it suffices to show not every fiber of $\widehat{P}$ is displaceable.

Suppose every fiber of $\widehat{P}$ is displaceable, then we can take an open cover $\left\{U_{j}\right\}_{j=1}^{d}$ of $\Delta$ such that each $\widehat{P}^{-1}\left(U_{j}\right) \subset V$ is displaceable. Since the coordinate functions of $P$ commute, for any two functions $f, g: \mathbb{R}^{n} \rightarrow \mathbb{R}$ the contactomorphisms $\widetilde{\phi}_{\widehat{P}^{*} f}$ and $\widetilde{\phi}_{\widehat{P}^{*} g}$ commute and $\widetilde{\phi}_{\widehat{P}^{*}(f+g)}=\widetilde{\phi}_{\widehat{P}^{*} f} \widetilde{\phi}_{\widehat{P}^{*} g}$. In particular if $\left\{f_{j}\right\}$ is a partition of unity subordinate to $\left\{U_{j}\right\}$, then

$$
\mu\left(\widetilde{\phi}_{1}\right)=\mu\left(\widetilde{\phi}_{\widehat{P}^{*} f_{1}}+\cdots+\widetilde{\phi}_{\widehat{P}^{*} f_{d}}\right)=\sum_{j=1}^{d} \mu\left(\widetilde{\phi}_{\widehat{P}^{*} f_{j}}\right)=0
$$


since homogeneous quasi-morphisms are homomorphisms when restricted to abelian subgroups and also that $\mu\left(\widetilde{\phi}_{\widehat{P}^{*} f_{j}}\right)=0$ by the vanishing property. However $\mu\left(\widetilde{\phi}_{1}\right)>0$, so we have a contradiction.

Remark 4.2. The proof of Theorem 1.17 also shows if there is monotone quasi-morphism $\mu$ : $\widetilde{\operatorname{Cont}}_{0}(V, \xi) \rightarrow \mathbb{R}$ with the vanishing property and $(V, \xi)$ is completely integrable contact manifold, in the sense of Khesin-Tabachnikov [KT10], then at least one of the pre-Lagrangian fibers is non-displaceable.

Let us now prove Proposition 1.13 which states that if a subheavy subset $Y \subset V$ is preserved by a positive contact vector field, then it is $\mu$-superheavy.

Proof of Proposition 1.13. We will assume that $Y$ is invariant under the flow for the Reeb vector field $R_{\alpha}$, since any positive contact vector field is the Reeb vector for some contact form [MS98, Chapter 3.4]. Given $h \in C^{\infty}(V)$ such that $\left.h\right|_{Y}>0$, since $Y$ is closed we have $\left.h\right|_{Y} \geq c$ for some positive $c \in \mathbb{R}$. Let $m$ be a positive integer and note that $\phi_{g}^{t}=\phi_{-m c}^{t} \phi_{m h}^{t}$ where

$$
g_{t}:=\alpha\left(-m c R_{\alpha}+d \phi_{-m c}^{t}\left(m X_{h}\right) \circ\left(\phi_{-m c}^{t}\right)^{-1}\right)=m\left(-c+h \circ \phi_{m c}^{t}\right) .
$$

Since $\phi_{m c}^{t}=\phi_{m c R_{\alpha}}^{t}$ is a reparametrization of the Reeb flow, which preserves $Y$, it follows that $\left.g_{t}\right|_{Y} \geq 0$ and hence $\mu\left(\widetilde{\phi}_{-m c} \widetilde{\phi}_{m h}\right)=\mu\left(\widetilde{\phi}_{g}\right) \geq 0$ since $\mu$ is monotone and $Y$ is $\mu$-subheavy. Since $h$ is autonomous it follows $\widetilde{\phi}_{m h}=\widetilde{\phi}_{h}^{m}$ and because $\mu$ is a homogeneous quasi-morphism we have

$$
m \mu\left(\widetilde{\phi}_{h}\right)=\mu\left(\widetilde{\phi}_{m h}\right) \geq \mu\left(\widetilde{\phi}_{-m c} \widetilde{\phi}_{m h}\right)+\mu\left(\widetilde{\phi}_{m c}\right)-D \geq \mu\left(\widetilde{\phi}_{m c}\right)-D=m \mu\left(\widetilde{\phi}_{c}\right)-D .
$$

By dividing through by $m$ and taking the limit as $m \rightarrow \infty$ gives $\mu\left(\widetilde{\phi}_{h}\right) \geq \mu\left(\widetilde{\phi}_{c}\right)$ and $\mu\left(\widetilde{\phi}_{c}\right)>0$ since $V$ is $\mu$-superheavy by Proposition 1.10 (iv).

\subsection{Proof of results from Section 1.3}

Here we will prove the results in Section 1.3 about the relation between quasi-morphisms on $\widetilde{\operatorname{Cont}}_{0}(V)$ and $\widetilde{\operatorname{Ham}}(M)$ when $\pi:(V, \alpha) \rightarrow(M, \omega)$ is a prequantization. Before proving Theorem 1.19 we need the following lemma.

Lemma 4.3. Let $\pi:(V, \alpha) \rightarrow(M, \omega)$ be a prequantization and let $\mu: \widetilde{\operatorname{Cont}}_{0}(V) \rightarrow \mathbb{R}$ be a monotone quasi-morphism, then

$$
\mu\left(\widetilde{\phi}_{c+\pi^{*} H}\right)=\left(\int_{0}^{1} c(t) d t\right) \mu\left(\widetilde{\phi}_{1}\right)+\mu\left(\widetilde{\phi}_{\pi^{*} H}\right)
$$

for all smooth functions $H:[0,1] \times M \rightarrow \mathbb{R}$ and $c:[0,1] \rightarrow \mathbb{R}$.

Proof. By using the contact Poisson bracket (1.3), or just the definitions, one sees that $\widetilde{\phi}_{c}$ and $\widetilde{\phi}_{\pi^{*} H}$ commute in $\widetilde{\operatorname{Cont}}_{0}(V)$ and $\widetilde{\phi}_{c+\pi^{*} H}=\widetilde{\phi}_{c} \widetilde{\phi}_{\pi^{*} H}$. Therefore since homogeneous quasimorphisms are homomorphisms on abelian subgroups

$$
\mu\left(\widetilde{\phi}_{c+\pi^{*} H}\right)=\mu\left(\widetilde{\phi}_{c}\right)+\mu\left(\widetilde{\phi}_{\pi^{*} H}\right)
$$


and hence it suffices to prove $\mu\left(\widetilde{\phi}_{c}\right)=\left(\int_{0}^{1} c(t) d t\right) \mu\left(\widetilde{\phi}_{1}\right)$.

Since $\widetilde{\phi}_{\kappa}=\widetilde{\phi}_{c}$ via a time-reparametrization where $\kappa=\int_{0}^{1} c(t) d t$, this reduces to proving $\mu\left(\widetilde{\phi}_{\kappa}\right)=\kappa \mu\left(\widetilde{\phi}_{1}\right)$ for all real numbers $\kappa \in \mathbb{R}$. For any integer $m \in \mathbb{Z}$, this holds since $\mu$ is homogeneous and $\widetilde{\phi}_{m}=\widetilde{\phi}_{1}^{m}$. This extends to rational numbers and since $\mu$ is monotone it then holds for all real scalars.

Proof of Theorem 1.19. Since $\pi^{*}: \widetilde{\operatorname{Ham}}(M) \rightarrow \widetilde{\operatorname{Cont}}_{0}(V)$ from 1.17) is a homomorphism it is clear that $\mu_{M}$ is a quasi-morphism. For stability let $c(t):=\min _{M}\left(H_{t}-G_{t}\right)$, then by monotonicity and Lemma 4.3 we have

$$
\left(\int_{0}^{1} c(t) d t\right) \mu\left(\widetilde{\phi}_{1}\right)+\mu\left(\widetilde{\phi}_{\pi^{*} G}\right)=\mu\left(\widetilde{\phi}_{c+\pi^{*} G}\right) \leq \mu\left(\widetilde{\phi}_{\pi^{*} H}\right)
$$

and hence

$$
\left(\int_{0}^{1} \min _{M}\left(H_{t}-G_{t}\right) d t\right) \mu\left(\widetilde{\phi}_{1}\right) \leq \mu\left(\widetilde{\phi}_{\pi^{*} H}\right)-\mu\left(\widetilde{\phi}_{\pi^{*} G}\right)
$$

After translating to the definition of $\mu_{M}$ in 1.18 this is the left-hand part of the stability condition (1.14). The right-hand side is proved analogously.

Lemma 4.3 shows that the formulas for $\zeta_{\mu_{M}}$ in $\sqrt{1.15}$ and Theorem 1.19 are equal. It follows from the formula in Theorem 1.19 that if $\mu$ has the vanishing property, then so does $\zeta_{\mu_{M}}$. This is because if $X \subset M$ is displaceable by an element of $\operatorname{Ham}(M)$, then $\pi^{-1}(X) \subset V$ is displaceable by an element of $\operatorname{Cont}_{0}(V)$. Going back to the quasi-morphism $\mu_{M}$, it follows from [Bor13, Proposition 1.7] that $\mu_{M}$ has the Calabi property if the associated quasi-state $\zeta_{\mu_{M}}$ has the vanishing property.

Proof of Proposition 1.20. For item (i), it is enough to show that if $H \in C^{\infty}(M)$ is such that $\left.H\right|_{\pi(Y)}=0$, then $\zeta_{\mu_{M}}(H)=0$. If $\left.H\right|_{\pi(Y)}=0$, then $\left.\pi^{*} H\right|_{Y}=0$ and $\mu\left(\widetilde{\phi}_{\pi^{*} H}\right)=0$ by the definition of $Y$ being $\mu$-subheavy. It then follows from Theorem 1.19 that $\zeta_{\mu_{M}}(H)=0$.

For item (ii), let $Y=\pi^{-1}(X)$ and let $h \in C^{\infty}(V)$ be such that $\left.h\right|_{Y}>0$. There is $H \in C^{\infty}(M)$ with $\pi^{*} H \leq h$ and $\left.H\right|_{X}>0$. From the monotonicity of $\mu$ and Theorem 1.19 we have

$$
\mu\left(\widetilde{\phi}_{h}\right) \geq \mu\left(\widetilde{\phi}_{\pi^{*} H}\right)=\mu\left(\widetilde{\phi}_{1}\right) \zeta_{\mu_{M}}(H)
$$

and therefore we are done since $\zeta_{\mu_{M}}(H) \geq \min _{X} H>0$ by the definition of $\zeta_{\mu_{M}}$-superheavy and since $\mu\left(\widetilde{\phi}_{1}\right)>0$ by Proposition 1.10 (iv).

\subsection{Proofs about Givental's quasi-morphism}

\subsubsection{A brief summary of Givental's quasi-morphism}

Recall that a point $v \in(V, \xi)$ in a contact manifold is a discriminant point for a contactomorphism $\phi \in \operatorname{Cont}(V, \xi)$ if

$$
\phi(v)=v \quad \text { and } \quad\left(\phi^{*} \alpha\right)_{v}=\alpha_{v}
$$

for some (and hence every) contact form $\alpha$ and the discriminant of $\operatorname{Cont}_{0}(V, \xi)$ is

$$
\Sigma(V, \xi):=\left\{\phi \in \operatorname{Cont}_{0}(V, \xi) \mid \phi \text { has at least one discriminant point }\right\} .
$$


A $C^{\infty}$-generic contactomorphism has no discriminant points. Indeed if $v$ is a discriminant point of $\phi$, then the image of $d \phi_{v}-\mathrm{id}_{T_{v} V}$ is contained in $\xi_{v}$ and hence $d \phi_{v}-\mathrm{id}_{T_{v} V}$ has a nontrivial kernel. This means $v$ is a degenerate fixed point and it is a standard fact that $C^{\infty}$-generic contactomorphisms do not have degenerate fixed points (see [HS95, Theorem 3.1] for a proof in the Hamiltonian case). In fact any $\phi \in \operatorname{Cont}_{0}(V)$ on the discriminant $\Sigma(V)$ can be perturbed off $\Sigma(V)$ via the Reeb flow, but we will not include the proof since this is not necessary for what follows.

In Giv90] Givental showed how to coorient the discriminant $\Sigma \subset \operatorname{Cont}_{0}\left(\mathbb{R} P^{2 d-1}\right)$ using generating functions. Given a smooth path $\gamma:[0, \tau] \rightarrow \operatorname{Cont}_{0}\left(\mathbb{R} P^{2 d-1}\right)$ with endpoints not on $\Sigma$, the coorientation gives a well-defined intersection index between $\gamma$ and $\Sigma$ denoted

$$
\mu^{G}(\gamma) \in \mathbb{Z}
$$

which Givental called the nonlinear Maslov index. From the intersection viewpoint, Givental specified [Giv90, Section 9] conventions so that $\mu^{G}$ is defined for all paths of contactomorphisms. Alternatively, as noted by Colin-Sandon [CS12, Section 7], the nonlinear Maslov index can be defined purely in terms of generating families, leading to a uniform definition of the nonlinear Maslov index for any smooth path of contactomorphisms of $\mathbb{R} P^{2 d-1}$. Here are some key properties of the nonlinear Maslov index:

(i) Given two paths $\gamma_{i}:\left[0, \tau_{i}\right] \rightarrow \operatorname{Cont}_{0}\left(\mathbb{R} P^{2 d-1}\right)$ with $\gamma_{0}\left(\tau_{0}\right)=\gamma_{1}(0)$, one has

$$
\mu^{G}\left(\gamma_{0}\right)+\mu^{G}\left(\gamma_{1}\right)=\mu^{G}\left(\gamma_{0} * \gamma_{1}\right)
$$

where $\gamma_{0} * \gamma_{1}:\left[0, \tau_{0}+\tau_{1}\right] \rightarrow \operatorname{Cont}_{0}\left(\mathbb{R} P^{2 d-1}\right)$ is their concatenation.

(ii) For any path $\gamma$ in $\operatorname{Cont}_{0}\left(\mathbb{R} P^{2 d-1}\right)$ and element $\phi \in \operatorname{Cont}_{0}\left(\mathbb{R} P^{2 d-1}\right)$

$$
\left|\mu^{G}(\gamma \phi)-\mu^{G}(\gamma)\right| \leq 2 d
$$

where $\gamma \phi$ is the path defined by $t \mapsto \gamma(t) \phi$.

(iii) If a path $\gamma$ in $\operatorname{Cont}_{0}\left(\mathbb{R} P^{2 d-1}\right)$ is disjoint from the discriminant, then

$$
\mu^{G}(\gamma)=0
$$

(iv) The nonlinear Maslov index $\mu^{G}(\gamma)$ is invariant under homotopies of $\gamma$ with fixed endpoints.

The first item follows from the construction as an intersection index, the second item is Giv90, Theorem 9.1(a)], and the final two properties are established by both Givental [Giv90, Section 9] and Colin-Sandon [CS12, Section 7].

If $\mathcal{P} \operatorname{Cont}_{0}\left(\mathbb{R} P^{2 d-1}\right)$ denotes the space of contact isotopies $\left\{\phi^{t}\right\}_{t \in[0,1]}$ with $\phi^{0}=\mathrm{id}$, then one defines the asymptotic nonlinear Maslov index to be

$$
\mu_{\text {Giv }}\left(\left\{\phi^{t}\right\}_{t \in[0,1]}\right):=\lim _{\tau \rightarrow \infty} \frac{\mu^{G}\left(\left\{\phi^{t}\right\}_{t \in[0, \tau]}\right)}{\tau}
$$

where $\left\{\phi^{t}\right\}_{t \in[0, \tau]}$ is given by concatenation so $\phi^{k+s}:=\phi^{s}\left(\phi^{1}\right)^{k}$ for $s \in[0,1]$ and $k \in \mathbb{N}$. Since $\mu^{G}$ is invariant under homotopies with fixed endpoints, the map in 4.5 descends to a map

$$
\mu_{\text {Giv }}: \widetilde{\operatorname{Cont}}_{0}\left(\mathbb{R} P^{2 d-1}\right) \rightarrow \mathbb{R}
$$


and this is the definition of Givental's quasi-morphism from 1.5. As a special case of 4.5 we have

$$
\mu_{\mathrm{Giv}}(\widetilde{\phi})=\lim _{m \rightarrow \infty} \frac{\mu^{G}\left(\widetilde{\phi}^{m}\right)}{m}
$$

for $\widetilde{\phi} \in \widetilde{\operatorname{Cont}_{0}}\left(\mathbb{R} P^{2 d-1}\right)$ and hence $\mu_{\text {Giv }}$ is homogeneous: $\mu_{\text {Giv }}\left(\widetilde{\phi}^{m}\right)=m \mu_{\text {Giv }}(\widetilde{\phi})$.

\subsubsection{A subheavy Legendrian}

Proof of Lemma 1.23. It suffices to prove $\mathbb{R} P_{L}^{d-1} \subset \mathbb{R} P^{2 d-1}$ is $\mu_{\text {Giv }}$-subheavy since it is preserved by radial projection 1.23 .

If $h$ is an autonomous contact Hamiltonian that vanishes on $\mathbb{R} P_{L}^{d-1}$, then $X_{h}$ is always tangent to $\mathbb{R} P_{L}^{d-1}$ since it is Legendrian. Therefore the Legendrian nonlinear Maslov index $\mu(\lambda)$ from [Giv90, Section 9] of the constant path of Legendrians $\lambda:=\left\{\phi_{h}^{t}\left(\mathbb{R} P_{L}^{d-1}\right)\right\}_{t \in[0, \tau]}$ vanishes. By the definition of $\mu_{\text {Giv }}$ in 4.5 and Giv90, Section 9, Corollary 2] we know

$$
\mu_{\mathrm{Giv}}\left(\widetilde{\phi}_{h}\right)=\lim _{\tau \rightarrow \infty} \frac{\mu^{G}\left(\left\{\phi_{h}^{t}\right\}_{t \in[0, \tau]}\right)}{\tau}=\lim _{\tau \rightarrow \infty} \frac{\mu\left(\left\{\phi_{h}^{t}\left(\mathbb{R} P_{L}^{d-1}\right)\right\}_{t \in[0, \tau]}\right)}{\tau}
$$

so $\mu_{\text {Giv }}\left(\widetilde{\phi}_{h}\right)=0$ and therefore $\mathbb{R} P_{L}^{d-1}$ is $\mu_{\text {Giv }}$-subheavy.

\subsubsection{Proving properties of Givental's quasi-morphism in Proposition $\mathbf{1 . 2}$}

Proof of Proposition 1.2 (Monotonicity). By [Giv90, Theorem 9.1(b)], or equivalently CS12, Lemma 7.6], we know $\mu^{G}(\widetilde{\phi}) \geq 0$ if $\widetilde{\phi} \succeq$ id, so it follows from 4.6 that

$$
0 \leq \mu_{\text {Giv }}(\widetilde{\phi}) \quad \text { if } \quad \text { id } \preceq \widetilde{\phi} .
$$

Now if $\widetilde{\phi} \preceq \widetilde{\psi}$, then id $\preceq \widetilde{\psi}^{m} \circ \widetilde{\phi}^{-m}$ and hence $\mu_{\text {Giv }}\left(\widetilde{\psi}^{m} \circ \widetilde{\phi}^{-m}\right) \geq 0$. Using this and that $\mu_{\text {Giv }}$ is a homogeneous quasi-morphism, we get

$$
m \mu_{\text {Giv }}(\widetilde{\psi})-m \mu_{\text {Giv }}(\widetilde{\phi})=\mu_{\text {Giv }}\left(\widetilde{\psi}^{m}\right)-\mu_{\text {Giv }}\left(\widetilde{\phi}^{m}\right) \geq \mu_{\text {Giv }}\left(\widetilde{\psi}^{m} \circ \widetilde{\phi}^{-m}\right)-D \geq-D .
$$

Dividing by $m$ and taking the limit $m \rightarrow \infty$, gives $\mu_{\text {Giv }}(\widetilde{\phi}) \leq \mu_{\text {Giv }}(\widetilde{\psi})$ and hence $\mu_{\text {Giv }}$ is monotone.

Proof of Proposition 1.2 ( $C^{0}$-continuity). Givental proved in Giv90, Corollary 3, Section 9] that $\mu_{\text {Giv }}$ is $C^{0}$-continuous for time-independent contact Hamiltonians and as he explained to us the proof generalizes to time-dependent contact Hamiltonians in the following way.

Suppose we have $C^{0}$-convergence $h^{(n)} \rightarrow h$ of contact Hamiltonians in $C^{\infty}\left([0,1] \times \mathbb{R} P^{2 d-1}\right)$. For a given $\epsilon>0$, pick an integer $m>0$ such that $6 d / m<\epsilon$ and by [Giv90, Theorem 9.1(c)] we know that if $n$ is sufficiently large, then

$$
\left|\mu^{G}\left(\left\{\phi_{h^{(n)}}^{t}\right\}_{t \in[0, m]}\right)-\mu^{G}\left(\left\{\phi_{h}^{t}\right\}_{t \in[0, m]}\right)\right| \leq 2 d .
$$

By (4.2) and 4.3), for any two integers $m, N>0$ and $k \in C^{\infty}\left([0,1] \times \mathbb{R} P^{2 d-1}\right)$ one has

$$
\left|\mu^{G}\left(\left\{\phi_{k}^{t}\right\}_{t \in[0, N m]}\right)-N \mu^{G}\left(\left\{\phi_{k}^{t}\right\}_{t \in[0, m]}\right)\right| \leq 2 d N
$$


which when applied to the previous inequality gives that if $n$ is sufficiently large, then

$$
\left|\mu^{G}\left(\left\{\phi_{h^{(n)}}^{t}\right\}_{t \in[0, N m]}\right)-\mu^{G}\left(\left\{\phi_{h}^{t}\right\}_{t \in[0, N m]}\right)\right| \leq 6 d N .
$$

Dividing by $N m$ and taking the limit as $N \rightarrow \infty$ gives

$$
\left|\mu_{\mathrm{Giv}}\left(\widetilde{\phi}_{h^{(n)}}\right)-\mu_{\mathrm{Giv}}\left(\widetilde{\phi}_{h}\right)\right| \leq \frac{6 d}{m}<\epsilon
$$

if $n$ is sufficiently large and therefore $\lim _{n \rightarrow \infty} \mu_{\mathrm{Giv}}\left(\widetilde{\phi}_{h^{(n)}}\right)=\mu_{\mathrm{Giv}}(\widetilde{\phi})$.

Proof of Proposition 1.2 (Vanishing property). For an open $U \subset \mathbb{R} P^{2 d-1}$ suppose there is a $\psi \in \operatorname{Cont}_{0}\left(\mathbb{R} P^{2 d-1}\right)$ such that $\psi(U) \cap U=\emptyset$ and without loss of generality we may assume $\psi$ has no discriminant points. By 4.2 we know

$$
\left|\mu^{G}\left(\left\{\phi_{h}^{t} \psi\right\}_{t \in[0, \tau]}\right)-\mu^{G}\left(\left\{\phi_{h}^{t}\right\}_{t \in[0, \tau]}\right)\right| \leq 2 d
$$

so if $\mu^{G}\left(\left\{\phi_{h}^{t} \psi\right\}_{t \in[0, \tau]}\right)=0$ for all $\tau \geq 0$, then it will follow from 4.5 that

$$
\mu_{\mathrm{Giv}}\left(\widetilde{\phi}_{h}\right)=\lim _{\tau \rightarrow \infty} \frac{\mu^{G}\left(\left\{\phi_{h}^{t}\right\}_{t \in[0, \tau]}\right)}{\tau}=0
$$

Therefore by (4.4) it remains to prove $\phi_{h}^{t} \psi$ has no discriminant points for all $t \geq 0$. Assume $p$ is a discriminant point for some $\phi_{h}^{t} \psi$. If $p \in U$, then $\psi(p)=\left(\phi_{h}^{t}\right)^{-1}(p) \in U$ but this contradicts that $\psi(U) \cap \bar{U}=\emptyset$. If $p \notin U$, then $\psi(p)=\left(\phi_{h}^{t}\right)^{-1}(p)=p$ so $p$ is a fixed point of $\psi$ and also a discriminant point of $\psi$, but we assumed they did not exist.

\section{References}

[AA12] G. Alston and L. Amorim. Floer cohomology of torus fibers and real Lagrangians in Fano toric manifolds. Int. Math. Res. Not. IMRN, 2012(12):2751-2793, 2012.

[Aar91] J. F. Aarnes. Quasi-states and quasi-measures. Adv. Math., 86(1):41-67, 1991.

[ABM14] M. Abreu, M. S. Borman, and D. McDuff. Displacing Lagrangian toric fibers by extended probes. Algebr. Geom. Topol., 14(2):687-752, 2014.

[AF13] P. Albers and U. Frauenfelder. A variational approach to Givental's nonlinear Maslov index. Geom. Funct. Anal., 23(1):482-499, 2013.

[AM12] M. Abreu and L. Macarini. Contact homology of good toric contact manifolds. Compos. Math., 148(1):304-334, 2012.

[AM13a] M. Abreu and L. Macarini. Remarks on Lagrangian intersections in toric manifolds. Trans. Amer. Math. Soc., 365(7):3851-3875, 2013.

[AM13b] P. Albers and W. J. Merry. Orderability, contact non-squeezing, and Rabinowitz Floer homology. arXiv:1302.6576, 2013. 
[Ban97] A. Banyaga. The structure of classical diffeomorphism groups, volume 400 of Mathematics and its Applications. Kluwer Academic Publishers Group, Dordrecht, 1997.

[Bat81] V. V. Batyrev. Toric Fano threefolds. Izv. Akad. Nauk SSSR Ser. Mat., 45(4):704-717, 927, 1981.

[Bat99] V. V. Batyrev. On the classification of toric Fano 4-folds. J. Math. Sci. (New York), 94(1):1021-1050, 1999.

[Bav91] C. Bavard. Longueur stable des commutateurs. Enseign. Math. (2), 37(1-2):109150, 1991.

[BC09a] P. Biran and O. Cornea. A Lagrangian quantum homology. In New perspectives and challenges in symplectic field theory, volume 49 of CRM Proc. Lecture Notes, pages 1-44. Amer. Math. Soc., Providence, RI, 2009.

[BC09b] P. Biran and O. Cornea. Rigidity and uniruling for Lagrangian submanifolds. Geom. Topol., 13(5):2881-2989, 2009.

[BEP04] P. Biran, M. Entov, and L. Polterovich. Calabi quasimorphisms for the symplectic ball. Commun. Contemp. Math., 6(5):793-802, 2004.

[BEP12] L. Buhovsky, M. Entov, and L. Polterovich. Poisson brackets and symplectic invariants. Selecta Math. (N.S.), 18(1):89-157, 2012.

[Bor12] M. S. Borman. Symplectic reduction of quasi-morphisms and quasi-states. J. Symplectic Geom., 10(2):225-246, 2012.

[Bor13] M. S. Borman. Quasi-states, quasi-morphisms, and the moment map. Int. Math. Res. Not. IMRN, 2013(11):2497-2533, 2013.

[Bra12] M. Brandenbursky. Quasi-morphisms and $L^{p}$-metrics on groups of volumepreserving diffeomorphisms. J. Topol. Anal., 4(2):255-270, 2012.

[BS07] G. Ben Simon. The nonlinear Maslov index and the Calabi homomorphism. Commun. Contemp. Math., 9(6):769-780, 2007.

[BS10] G. Ben Simon. The geometry of partial order on contact transformations of prequantization manifolds. In Arithmetic and geometry around quantization, volume 279 of Progr. Math., pages 37-64. Birkhäuser Boston Inc., Boston, MA, 2010 .

[BS13] M. Brandenbursky and E. Shelukhin. On the large-scale geometry of the $L^{p_{-}}$ metric on the symplectomorphism group of the two-sphere. arXiv:1304.7037v1, 2013.

[BSH11] G. Ben Simon and T. Hartnick. Quasi-total orders and translation numbers. arXiv:1106.630\%, 2011. 
[BSH12] G. Ben Simon and T. Hartnick. Reconstructing quasimorphisms from associated partial orders and a question of Polterovich. Comment. Math. Helv., 87(3):705$725,2012$.

[BW58] W. M. Boothby and H. C. Wang. On contact manifolds. Ann. of Math. (2), 68:721-734, 1958.

[Cal09] D. Calegari. scl, volume 20 of MSJ Memoirs. Mathematical Society of Japan, Tokyo, 2009.

[Che96] Yu. V. Chekanov. Critical points of quasifunctions, and generating families of Legendrian manifolds. Funktsional. Anal. i Prilozhen., 30(2):56-69, 96, 1996.

[Cho04] C.-H. Cho. Holomorphic discs, spin structures, and Floer cohomology of the Clifford torus. Int. Math. Res. Not., 2004(35):1803-1843, 2004.

[Cho08] C.-H. Cho. Non-displaceable Lagrangian submanifolds and Floer cohomology with non-unitary line bundle. J. Geom. Phys., 58(11):1465-1476, 2008.

[CN10] V. Chernov and S. Nemirovski. Non-negative Legendrian isotopy in $S T^{*} M$. Geom. Topol., 14(1):611-626, 2010.

[CP12] C.-H. Cho and M. Poddar. Holomorphic orbidiscs and Lagrangian Floer cohomology of symplectic toric orbifolds. arXiv:1206.3994v4, 2012.

[CS12] V. Colin and S. Sandon. The discriminant and oscillation lengths for contact and Legendrian isotopies. J. Eur. Math. Soc. (JEMS) (to appear), arXiv:1205.2102, 2012.

[Del88] T. Delzant. Hamiltoniens périodiques et images convexes de l'application moment. Bull. Soc. Math. France, 116(3):315-339, 1988.

[EHS95] Y. Eliashberg, H. Hofer, and D. Salamon. Lagrangian intersections in contact geometry. Geom. Funct. Anal., 5(2):244-269, 1995.

[EKP06] Y. Eliashberg, S. S. Kim, and L. Polterovich. Geometry of contact transformations and domains: orderability versus squeezing. Geom. Topol., 10:1635-1747, 2006.

[Eli91] Y. Eliashberg. New invariants of open symplectic and contact manifolds. J. Amer. Math. Soc., 4(3):513-520, 1991.

[Ent04] M. Entov. Commutator length of symplectomorphisms. Comment. Math. Helv., 79(1):58-104, 2004.

[EP00] Y. Eliashberg and L. Polterovich. Partially ordered groups and geometry of contact transformations. Geom. Funct. Anal., 10(6):1448-1476, 2000.

[EP03] M. Entov and L. Polterovich. Calabi quasimorphism and quantum homology. Int. Math. Res. Not., 2003(30):1635-1676, 2003. 
[EP06] M. Entov and L. Polterovich. Quasi-states and symplectic intersections. Comment. Math. Helv., 81(1):75-99, 2006.

[EP08] M. Entov and L. Polterovich. Symplectic quasi-states and semi-simplicity of quantum homology. In Toric topology, volume 460 of Contemp. Math., pages 47-70. Amer. Math. Soc., Providence, RI, 2008.

[EP09a] M. Entov and L. Polterovich. $C^{0}$-rigidity of the double Poisson bracket. Int. Math. Res. Not. IMRN, 2009(6):1134-1158, 2009.

[EP09b] M. Entov and L. Polterovich. Rigid subsets of symplectic manifolds. Compos. Math., 145(3):773-826, 2009.

[EP10] M. Entov and L. Polterovich. $C^{0}$-rigidity of Poisson brackets. In Symplectic topology and measure preserving dynamical systems, volume 512 of Contemp. Math., pages 25-32. Amer. Math. Soc., Providence, RI, 2010.

[EPP12] M. Entov, L. Polterovich, and P. Py. On continuity of quasimorphisms for symplectic maps. In Perspectives in analysis, geometry, and topology, volume 296 of Progr. Math., pages 169-197. Birkhäuser/Springer, New York, 2012.

[EPZ07] M. Entov, L. Polterovich, and F. Zapolsky. Quasi-morphisms and the Poisson bracket. Pure Appl. Math. Q., 3(4, Special Issue: In honor of Grigory Margulis. Part 1):1037-1055, 2007.

[FOOO09] K. Fukaya, Y.-G. Oh, H. Ohta, and K. Ono. Lagrangian intersection Floer theory: anomaly and obstruction. Part I-II, volume 46 of AMS/IP Studies in Advanced Mathematics. American Mathematical Society, Providence, RI, 2009.

[FOOO11a] K. Fukaya, Y.-G. Oh, H. Ohta, and K. Ono. Lagrangian Floer theory on compact toric manifolds II: bulk deformations. Selecta Math. (N.S.), 17(3):609-711, 2011.

[FOOO11b] K. Fukaya, Y.-G. Oh, H. Ohta, and K. Ono. Spectral invariants with bulk quasimorphisms and Lagrangian Floer theory. arXiv:1105.5123v2, 2011.

[FPR12] M. Fraser, L. Polterovich, and D. Rosen. On Sandon-type metrics for contactomorphism groups. arXiv:120\%.3151, 2012.

[Gei97] H. Geiges. Constructions of contact manifolds. Math. Proc. Cambridge Philos. Soc., 121(3):455-464, 1997.

[GG04] J.-M. Gambaudo and É. Ghys. Commutators and diffeomorphisms of surfaces. Ergodic Theory Dynam. Systems, 24(5):1591-1617, 2004.

[Ghy01] É. Ghys. Groups acting on the circle. Enseign. Math. (2), 47(3-4):329-407, 2001.

[Ghy07] É. Ghys. Knots and dynamics. In International Congress of Mathematicians. Vol. I, pages 247-277. Eur. Math. Soc., Zürich, 2007.

[Giv90] A. B. Givental. Nonlinear generalization of the Maslov index. In Theory of singularities and its applications, volume 1 of Adv. Soviet Math., pages 71-103. Amer. Math. Soc., Providence, RI, 1990. 
[GKS12] S. Guillermou, M. Kashiwara, and P. Schapira. Sheaf quantization of Hamiltonian isotopies and applications to nondisplaceability problems. Duke Math. J., 161(2):201-245, 2012.

[Hau13] L. Haug. On the quantum homology of real Lagrangians in Fano toric manifolds. Int. Math. Res. Not. IMRN, 2013(14):3171-3220, 2013.

[HS95] H. Hofer and D. A. Salamon. Floer homology and Novikov rings. In The Floer memorial volume, volume 133 of Progr. Math., pages 483-524. Birkhäuser, Basel, 1995.

[Kha09] M. Khanevsky. Hofer's metric on the space of diameters. J. Topol. Anal., 1(4):407-416, 2009.

[Kot04] D. Kotschick. What is... a quasi-morphism? Notices Amer. Math. Soc., 51(2):208-209, 2004.

[Kot08] D. Kotschick. Stable length in stable groups. In Groups of diffeomorphisms, volume 52 of Adv. Stud. Pure Math., pages 401-413. Math. Soc. Japan, Tokyo, 2008.

[KPS13] Y. Karshon, M. Pabiniak, and S. Sandon. Private communication. 2013.

[KT10] B. Khesin and S. Tabachnikov. Contact complete integrability. Regul. Chaotic Dyn., 15(4-5):504-520, 2010.

[Lan11] S. Lanzat. Quasi-morphisms and symplectic quasi-states for convex symplectic manifolds. Int. Math. Res. Not. IMRN (to appear), arXiv:1110.1555, 2011.

[Lan13a] S. Lanzat. Hamiltonian Floer homology for compact convex symplectic manifolds. arXiv:1302.1025, 2013.

[Lan13b] S. Lanzat. Quantum homology of compact convex symplectic manifolds. arXiv:1302.1021, 2013.

[Lau86] F. Laudenbach. Homotopie régulière inactive et engouffrement symplectique. Ann. Inst. Fourier (Grenoble), 36(2):93-111, 1986.

[Lu08] G. Lu. Symplectic fixed points and Lagrangian intersections on weighted projective spaces. Houston J. Math., 34(1):301-316, 2008.

[McD11] D. McDuff. Displacing Lagrangian toric fibers via probes. In Low-dimensional and symplectic topology, volume 82 of Proc. Sympos. Pure Math., pages 131-160. Amer. Math. Soc., Providence, RI, 2011.

[Mil08] I. Milin. Orderability of contactomorphism groups of lens spaces. ProQuest LLC, Ann Arbor, MI, 2008. Thesis (Ph.D.)-Stanford University.

[Mor01] S. Morita. Geometry of differential forms, volume 201 of Translations of Mathematical Monographs. American Mathematical Society, Providence, RI, 2001. Translated from the two-volume Japanese original $(1997,1998)$ by Teruko Nagase and Katsumi Nomizu, Iwanami Series in Modern Mathematics. 
[MS98] D. McDuff and D. Salamon. Introduction to symplectic topology. Oxford Mathematical Monographs. The Clarendon Press Oxford University Press, New York, second edition, 1998.

[MT10] D. McDuff and S. Tolman. Polytopes with mass linear functions. I. Int. Math. Res. Not. IMRN, 2010(8):1506-1574, 2010.

[MVZ12] A. Monzner, N. Vichery, and F. Zapolsky. Partial quasimorphisms and quasistates on cotangent bundles, and symplectic homogenization. J. Mod. Dyn., 6(2):205-249, 2012.

[Øbr07] M. Øbro. An algorithm for the classification of smooth Fano polytopes. arXiv:0704.0049, 2007.

[Ono96] K. Ono. Lagrangian intersection under Legendrian deformations. Duke Math. J., 85(1):209-225, 1996.

[Ost06] Y. Ostrover. Calabi quasi-morphisms for some non-monotone symplectic manifolds. Algebr. Geom. Topol., 6:405-434 (electronic), 2006.

[Pol06] L. Polterovich. Floer homology, dynamics and groups. In Morse theoretic methods in nonlinear analysis and in symplectic topology, volume 217 of NATO Sci. Ser. II Math. Phys. Chem., pages 417-438. Springer, Dordrecht, 2006.

[Py06] P. Py. Quasi-morphismes et invariant de Calabi. Ann. Sci. École Norm. Sup. (4), 39(1):177-195, 2006.

[Ryb10] T. Rybicki. Commutators of contactomorphisms. Adv. Math., 225:3291-3326, 2010.

[San10] S. Sandon. An integer-valued bi-invariant metric on the group of contactomorphisms of $\mathbb{R}^{2 n} \times S^{1}$. J. Topol. Anal., 2(3):327-339, 2010 .

[San11a] S. Sandon. Contact homology, capacity and non-squeezing in $\mathbb{R}^{2 n} \times S^{1}$ via generating functions. Ann. Inst. Fourier (Grenoble), 61(1):145-185, 2011.

[San11b] S. Sandon. Equivariant homology for generating functions and orderability of lens spaces. J. Symplectic Geom., 9(2):123-146, 2011.

[Sat00] H. Sato. Toward the classification of higher-dimensional toric Fano varieties. Tohoku Math. J. (2), 52(3):383-413, 2000.

[She11] E. Shelukhin. The action homomorphism, quasimorphisms and moment maps on the space of compatible almost complex structures. arXiv:1105.5814v2, 2011.

[Tam08] D. Tamarkin. Microlocal condition for non-displaceability. arXiv:0809.1584, 2008.

[Ush11] M. Usher. Deformed Hamiltonian Floer theory, capacity estimates, and Calabi quasimorphisms. Geom. Topol., 15(3):1313-1417, 2011. 
[Woo11] C. T. Woodward. Gauged Floer theory of toric moment fibers. Geom. Funct. Anal., 21(3):680-749, 2011.

[WW82] K. Watanabe and M. Watanabe. The classification of Fano 3-folds with torus embeddings. Tokyo J. Math., 5(1):37-48, 1982.

[WW13] G. Wilson and C. T. Woodward. Quasimap Floer cohomology for varying symplectic quotients. Canad. J. Math., 65(2):467-480, 2013.

[Zap13] F. Zapolsky. Geometry of contactomorphism groups, contact rigidity, and contact dynamics in jet spaces. Int. Math. Res. Not. IMRN, 2013(20):4687-4711, 2013.

\author{
Matthew Strom Borman Frol Zapolsky \\ Stanford University \\ University of Haifa \\ borman@stanford.edu \\ frol.zapolsky@gmail.com
}

